\title{
Some New Traveling Wave Solutions of Modified Camassa Holm Equation by the Improved G'/G Expansion Method
}

\author{
Rida Tassew Redi*, Akalu Abriham Anulo \\ Department of Mathematics, Institute of Technology, Dire Dawa University, Dire Dawa, Ethiopia \\ Email address: \\ tassew0@gmail.com(R. T.Redi), ake.abrish@gmail.com(A. A. Anulo) \\ ${ }^{*}$ Corresponding author \\ To cite this article: \\ Rida Tassew Redi, Akalu Abriham Anulo. Some New Traveling Wave Solutions of Modified Camassa Holm Equation by the Improved \\ G'/G Expansion Method. Mathematics and Computer Science. Vol. 3, No. 1, 2018, pp. 23-45. doi: 10.11648/j.mcs.20180301.14
}

Received: January 17, 2018; Accepted: February 16, 2018; Published: April 9, 2018

\begin{abstract}
In this article, the improved $\mathrm{G}^{\prime} / \mathrm{G}$-expansion method has been implemented to generate travelling wave solutions, where $G(\eta)$ satisfies the second order nonlinear ordinary differential equation. To show the advantages of the method, the Simplified Modified Camassa Holm ( $\mathrm{SMCH}$ ) equation has been investigated. Nonlinear partial differential equations have many potential applications in mathematical physics and engineering sciences. Some of our solutions are in good agreement with already published results for a special case and others are new. The solutions in this work may express a variety of new features of waves. Furthermore, these solutions can be valuable in the theoretical and numerical studies of the considered equation.
\end{abstract}

Keywords: Improved G'/G-Expansion Method, The SMCH Equation, Traveling Wave Solutions, Nonlinear Evolution Equations

\section{Introduction}

Nonlinear partial differential equations (NLPDEs) are widely used to describe complex physical phenomena arising in scientific and engineering fields, such as, plasma physics, fluid mechanics, solid state physics, quantum mechanics, nonlinear optics, chemical physics and many others. Exact travelling wave solutions of NLPDEs play an important role in the study of nonlinear physical phenomena. Looking for exact solutions to nonlinear evolution equations (NLEEs) has long been a major concern for both mathematicians and physicists. These solutions may well describe various phenomena in physics and other fields [1].But unlike LPDE, NPDEs are difficult to study because there are almost no general techniques that work for all NPDEs, and usually each individual equation has to be studied as a separate problem. Therefore, many authors have been introducing different techniques to obtain exact traveling wave solutions for nonlinear evolution equations (NLEEs) for the past many years. Recently, several direct methods such as, the Backlund transform method [1], the variational iteration method [2], Hirotas bi-linear methods [3], the sine-cosine function method [4-5], Jacobi elliptic method [6], the standard tanh and extended tanh methods [7-11], the exp-function method [12-13], the inverse scattering method [14] and others have been proposed to obtain exact solutions of nonlinear partial differential equations. Using these methods many exact solutions, including the solitary wave solutions, shock wave solutions and periodic wave solutions are obtained for some kinds of nonlinear evolution equations.

Another important method presented to construct exact solutions of nonlinear PDEs is the basic $\left(G^{\prime} / G\right)$-expansion method. The concept of this method was first proposed by Wang et al. [15], consequently, many researchers applied the $\left(G^{\prime} / G\right)$-expansion method to solve different kinds of NLEEs [16-19]. More recently, Zhang et al. [20] extended the basic $\left(G^{\prime} / G\right)$-expansion method which is called the improved $\left(G^{\prime} / G\right)$-expansion method to establish abundant traveling wave solutions of nonlinear PDEs. Many researchers applied the improved $\left(G^{\prime} / G\right)$-expansion method to different nonlinear PDEs [21-26f].It has been shown that this method is straightforward, concise, basic and effective.

Many researchers studied the Simplified Modified Camassa Holm $(\mathrm{MCH})$ by using different methods for 
constructing traveling wave solutions. For instance, Wazwaz [27] examined this equation to obtain exact solutions by using the sine-cosine algorithm. Liu et al. [28] implemented Wang's $\left(G^{\prime} / G\right)$-expansion method to establish analytical solutions. Recently, Hassibun Naher et al. [29] examined this equation to obtain exact solutions by using improved $\left(G^{\prime} / G\right)$ -expansion method where $G=G(\eta) \quad$ satisfies $G^{\prime \prime}+\lambda G^{\prime}+\mu G=0$.

The importance of our current work is, in order to generate abundant traveling wave solutions of the Simplified Modified Camassa Holm (MCH ) equation has been considered by applying the improved $\left(G^{\prime} / G\right)$-expansion method where $G=G(\eta) \quad$ satisfies $\quad G G^{\prime \prime}=\lambda G G^{\prime}+\mu G^{2}+V\left(G^{\prime}\right)^{2} \quad$. The structure of this paper is organized as follows. In section 2 , the improved $\left(G^{\prime} / G\right)$-expansion method is discussed. Application of improved $\left(G^{\prime} / G\right)$-expansion method to the Simplified Modified Camassa Holm $(\mathrm{MCH})$ is presented in Section 3. In Section 4 ends this work with a brief conclusion.

\section{The Improved ( $\left.G^{\prime} / G\right)$-Expansion Method}

We consider that the given Nonlinear Partial Differential Equation in the form of

$$
P\left(u, u_{x}, u_{t}, u_{x t}, u_{x x}, \ldots\right)=0
$$

where $\mathrm{P}$ is a polynomial in its arguments, which includes nonlinear terms and the highest order derivatives, the subscript stands for partial derivatives and $u(x, t)$ is the unknown function.

REMARK1: Nonlinear evolution equation (NLEE) is a NPDE which is dependent of a time t.

Travelling Wave Solution

A travelling wave solution of a NPDE in two variables $(x, t)$ is a solution of the form

$u(x, t)=U(\eta),(\eta=x-c t, \mathrm{c}$ is a speed of traveling wave) where $U(\eta)$ is an arbitrary di erentiable function of $\eta$.

The traveling wave transformations
Combining the real variables $x$ and $t$ by a wave variable $\eta=k x+\omega t$

$$
u(x, t)=U(\eta)
$$

where $\omega$ is the speed of the traveling wave.

The traveling wave transformations (2) converts (1) into an ordinary differential equation (ODE)

$$
Q\left(U, k U^{\prime}, \omega U^{\prime}, k \omega U^{\prime \prime}, k^{2} U^{\prime \prime}, \ldots\right)=0
$$

Where $Q$ is a polynomial in $U$ and its derivatives; the superscripts indicate the ordinary derivatives with respect to $\eta$.

Traveling wave solutions

The solution of (3) can be expressed as follows:

$$
U(\eta)=\sum_{i=0}^{m} \alpha_{i}\left(\mathrm{G}^{\prime} / \mathrm{G}\right)^{i}+\sum_{i=1}^{m} \beta_{i}\left(\mathrm{G}^{\prime} / \mathrm{G}\right)^{-i}
$$

Where $\alpha_{i}(i=0,1,2, \ldots, m), \quad \beta_{i}(i=1,2, \ldots, m)$ are arbitrary constants to be determined and either $\alpha_{m}$ or $\beta_{m}$ can be zero but both can't be zero at the same time [21] and $G=G(\eta)$ satisfies the following second order nonlinear ordinary differential equation with constant coefficients:

$$
G G^{\prime \prime}=\lambda G G^{\prime}+\mu G^{2}+V\left(G^{\prime}\right)^{2}
$$

Where the prime stands for derivative with respect to $\eta$ and $\lambda, \mu$, and $V$ are real parameters.

The Cole-Hops transformation $\phi(\eta)=\ln (G(\eta))_{\eta}$ transforms (5) into the generalized Riccati type equation in terms of $\phi(\eta)$ :

$$
\phi^{\prime}(\eta)=\mu+\lambda \phi(\eta)+(v-1) \phi^{2}(\eta)
$$

where $\phi(\eta)=\left(G^{\prime}(\eta) / G(\eta)\right)$. The generalized Riccati equation has 25 distinct solutions [24] and (see Appendix I for details)

Note that from (4), (5) and (6) it follows:

$$
\begin{gathered}
U^{\prime}(\eta)=\left(\mu+\lambda\left(\mathrm{G}^{\prime} / \mathrm{G}\right)+(V-1)\left(\mathrm{G}^{\prime} / \mathrm{G}\right)^{2}\right)\left(\sum_{i=0}^{m} i \alpha_{i}\left(\mathrm{G}^{\prime} / \mathrm{G}\right)^{i-1}-\sum_{i=1}^{m} i \beta_{i}\left(\mathrm{G}^{\prime} / \mathrm{G}\right)^{-i-1}\right) \\
U^{\prime \prime}(\eta)=\left(\mu+\lambda\left(\mathrm{G}^{\prime} / \mathrm{G}\right)+(V-1)\left(\mathrm{G}^{\prime} / \mathrm{G}^{2}\right)\left(\lambda+2(V-1)\left(\mathrm{G}^{\prime} / \mathrm{G}\right)\right)\left(\sum_{i=0}^{m} i \alpha_{i}\left(\mathrm{G}^{\prime} / \mathrm{G}\right)^{i-1}-\sum_{i=1}^{m} i \beta_{i}\left(\mathrm{G}^{\prime} / \mathrm{G}\right)^{-i-1}\right)-\right. \\
\left(\mu+\lambda\left(\mathrm{G}^{\prime} / \mathrm{G}\right)+(V-1)\left(\mathrm{G}^{\prime} / \mathrm{G}\right)^{2}\right)\left(\sum_{i=0}^{m} i(i-1) \alpha_{i}\left(\mathrm{G}^{\prime} / \mathrm{G}\right)^{i-2}\right)
\end{gathered}
$$

And so forth, where the prime denotes derivative with respect to $\eta$.
Now, to determine $u(x, t)$ explicitly we follow the following steps: 
Step 1: transforming (1) into (3) (ODE) using traveling wave transformations in (2).

Step 2: substitute (4) including (6) and (7) into (3) to determine the positive integer $\mathrm{m}$, taking the homogeneous balance between the highest order nonlinear term and the derivative of the highest order appearing in (3).

Step 3: Substitute (4) including (6) and (7) into (3) with the value of $\mathrm{m}$ obtained in Step 2, to obtain polynomials in $\left(G^{\prime} / G\right)^{i}(i=0,1, \ldots, m)$ and $\left(G^{\prime} / G\right)^{-i}(i=1,2, \ldots, m)$

subsequently, we collect each coefficient of the resulted polynomials to zero, yields a set of algebraic equations for $\alpha_{0}, \alpha_{1}, \ldots \alpha_{m}, \beta_{1}, \ldots \beta_{m}$, $\omega$ and $\mu$.

Step 4: Suppose that the value of the constants $\alpha_{i}(i=01, \ldots m), \beta_{i}(i=1,2, \ldots m), \mu$ and $\omega$ can be found by solving the algebraic equations obtained in Step 5. Since the general solutions of (6) are known to us, inserting the values of $\alpha_{i}(i=01,2, \ldots m), \beta_{i}(i=1,2, \ldots m), \mu$ and $\omega$ into (4), we obtain more general type and new exact traveling wave solutions of the nonlinear partial differential equation (1).

\section{Application of the Method}

In this section, the simplified $\mathrm{MCH}$ equation has been studied to obtain more general and new abundant traveling wave solutions including solitons, periodic and rational solutions by applying the improve $\left(G^{\prime} / G\right)$-expansion method.

Now considering the Simplified Modified Camassa Holm (MCH) [23]:

$$
u_{t}+2 k u_{x}-u_{x x t}+\beta u^{2} u_{x}=0, k \in \mathbb{R} \text { and } \beta>0
$$

To look for travelling wave solutions of (8), we use the wave transformation (2) and change (3) into the form of an ODE

$$
(2 k-\omega) U+\omega k U^{\prime \prime}+\frac{\beta}{3} U^{3}+A=0
$$

Now the homogeneous balance between $U^{\prime \prime}$ and $U^{3}$ in (9) can be found from

$$
U^{\prime \prime}=U^{3} \Rightarrow m+2=3 m \Rightarrow m=1
$$

Therefore, (4) becomes

$$
U(\xi)=\alpha_{0}+\alpha_{1}\left(\frac{G^{\prime}}{G}\right)+\beta_{1}\left(\frac{G^{\prime}}{G}\right)^{-1} \alpha_{1} \neq 0 \text { or } \beta_{1} \neq 0
$$

Now inserting (10) and its first and second derivative with (5) and (6) into (9) we obtained polynomials in $\left(G^{\prime} / G\right)^{i}(i=0,1,2, \ldots m)$ and $\left(G^{\prime} / G\right)^{-i}(i=1,2,3, \ldots m)$. Subsequently, we collect each coefficient of the resulted polynomials to zero, yields a set of algebraic equations in for $\alpha_{0}, \alpha_{1}, \beta_{1}, \alpha_{2}, \beta_{2}$ and $F$ (which we do not include it here for simplicity), so Solving the systems of obtained algebraic equations with the aid of algebraic software Maple 17, we obtain the following four different sets of values:

Case-1:

$$
\begin{aligned}
& A=0, \omega=\frac{4 k}{p^{2}-4 q(v-1)+2}, \alpha_{0}=\mp p \frac{\sqrt{6 k}}{\sqrt{\beta\left(4 q(v-1)-2-p^{2}\right)}}, \alpha_{1}=0, \\
& \beta_{1}= \pm 2 q \sqrt{\frac{6 k}{\beta\left(4 q(v-1)-2-p^{2}\right)}}
\end{aligned}
$$

Case-2

$$
\begin{aligned}
& A=0, \alpha_{0}=\mp p \frac{\sqrt{6 k}}{\sqrt{\beta\left(4 q(v-1)-2+p^{2}\right)}}, \alpha_{1}=\mp \frac{2 \sqrt{6 k}(v-1)}{\sqrt{\beta\left(4 q(v-1)-2+p^{2}\right)}}, \beta_{1}=0 \\
& \omega=\frac{-4 k}{4 q(v-1)-2-p^{2}},
\end{aligned}
$$

Case-3

$$
\begin{aligned}
& A= \pm \frac{16 \sqrt{6} i(-p q)(v-1) k^{\frac{3}{2}}}{\left(p^{2}+8 q(v-1)+2\right)^{\frac{3}{2}} \sqrt{\beta}}, \omega=\frac{4 k}{p^{2}+8 q(v-1)+2}, \alpha_{0}=\mp \frac{p i \sqrt{6 k}}{\sqrt{\beta\left(p^{2}+8 q(v-1)+2\right)}}, \\
& \beta_{1}=\mp \frac{2 q i \sqrt{6 k}}{\sqrt{\beta\left(p^{2}+8 q(v-1)+2\right)}} \alpha_{1}=\mp \frac{2 i \sqrt{6 k}(v-1)}{\sqrt{\beta\left(p^{2}+8 q(v-1)+2\right)}}
\end{aligned}
$$

Substituting (11) - (13) into (10) we obtain respectively: 


$$
\begin{gathered}
u_{1}(x, t)=\left(\mp p \frac{\sqrt{6 k}}{\sqrt{4 q \beta(v-1)-2 \beta-\beta p^{2}}} \pm 2 q \sqrt{\frac{6 k}{4 q \beta(v-1)-2 \beta-\beta p^{2}}}\left(\frac{G^{\prime}}{G}\right)^{-1}\right) \\
u_{2}(x, t)=\left(\mp p \frac{\sqrt{6 k}}{\sqrt{4 q \beta(v-1)-2 \beta+\beta p^{2}}} \mp \frac{2 \sqrt{6 k}(v-1)}{\sqrt{4 q \beta(v-1)-2 \beta+\beta p^{2}}}\left(\frac{G^{\prime}}{G}\right)\right) \\
u_{3}(x, t)=\left(\begin{array}{l}
\left.\mp \frac{p i \sqrt{6 k}}{\sqrt{\beta\left(p^{2}+8 q(v-1)+2\right)}} \mp \frac{2 i \sqrt{6 k}(v-1)}{\sqrt{\beta\left(p^{2}+8 q(v-1)+2\right)}}\left(\frac{G^{\prime}}{G}\right) \mp\right) \\
\sqrt{\beta\left(p^{2}+8 q(\sqrt{6 k}\right.}
\end{array}\right)
\end{gathered}
$$

Substituting the solutions of the (6) (see appendix I) into (14) and simplifying we obtain the following solutions of our target equation (8)

when $\Omega=p^{2} ? 4 q(v-1)>0$ and $p(v-1) \neq 0($ or $q(v-1) \neq 0)$

$$
\begin{aligned}
& u_{1_{1}}(x, t)=\left(\begin{array}{l}
\mp p \frac{\sqrt{6 k}}{\sqrt{\beta\left(4 q(v-1)-2-p^{2}\right)}} \pm 2 q \sqrt{\frac{6 k}{\beta\left(4 q(v-1)-2-p^{2}\right)}} \\
\left(-\frac{1}{2(v-1)}\left(p+\sqrt{\Omega} \tanh \left(\frac{1}{2} \sqrt{\Omega} \eta\right)\right)\right)^{-1}
\end{array}\right) \\
& u_{1_{2}}(x, t)=\left(\begin{array}{l}
\mp p \frac{\sqrt{6 k}}{\sqrt{\beta\left(4 q(v-1)-2-p^{2}\right)}} \pm 2 q \sqrt{\frac{6 k}{\beta\left(4 q(v-1)-2-p^{2}\right)}} \\
\left(-\frac{1}{2(v-1)}\left(p+\sqrt{\Omega} \operatorname{coth}\left(\frac{1}{2} \sqrt{\Omega} \eta\right)\right)\right)^{-1}
\end{array}\right) \\
& u_{1_{3}}(x, t)=\left(\begin{array}{l}
\mp p \frac{\sqrt{6 k}}{\sqrt{\beta\left(4 q(v-1)-2-p^{2}\right)}} \pm 2 q \sqrt{\frac{6 k}{\beta\left(4 q(v-1)-2-p^{2}\right)}} \\
\left(-\frac{1}{2(v-1)}\left(p+\sqrt{\Omega}\left(\tanh \left(\frac{1}{2} \sqrt{\Omega} \eta\right) \pm i \sec h\left(\frac{1}{2} \sqrt{\Omega} \eta\right)\right)\right)\right)^{-1}
\end{array}\right) \\
& u_{1_{4}}(x, t)=\left(\begin{array}{l}
\mp p \frac{\sqrt{6 k}}{\sqrt{\beta\left(4 q(v-1)-2-p^{2}\right)}} \pm 2 q \sqrt{\frac{6 k}{\beta\left(4 q(v-1)-2-p^{2}\right)}} \\
\left(-\frac{1}{2(v-1)}\left(p+\sqrt{\Omega}\left(\operatorname{coth}\left(\frac{1}{2} \sqrt{\Omega} \eta\right) \pm \operatorname{csch}\left(\frac{1}{2} \sqrt{\Omega} \eta\right)\right)\right)\right)^{-1}
\end{array}\right) \\
& u_{1_{5}}(x, t)=\left(\begin{array}{l}
\mp p \frac{\sqrt{6 k}}{\sqrt{\beta\left(4 q(v-1)-2-p^{2}\right)}} \pm 2 q \sqrt{\frac{6 k}{\beta\left(4 q(v-1)-2-p^{2}\right)}} \\
\left(-\frac{1}{4(v-1)}\left(2 p+\sqrt{\Omega}\left(\tanh \left(\frac{1}{4} \sqrt{\Omega} \eta\right)+\operatorname{coth}\left(\frac{1}{4} \sqrt{\Omega} \eta\right)\right)\right)\right)^{-1}
\end{array}\right)
\end{aligned}
$$




$$
\begin{aligned}
& u_{1_{6}}(x, t)=\left(\begin{array}{l}
\mp p \frac{\sqrt{6 k}}{\sqrt{\beta\left(4 q(v-1)-2-p^{2}\right)}} \pm 2 q \sqrt{\frac{6 k}{\beta\left(4 q(v-1)-2-p^{2}\right)}} \\
\left(\frac{1}{2(v-1)}\left(-p+\frac{ \pm \sqrt{\Omega\left(A^{2}-B^{2}\right)}-A \sqrt{\Omega} \cosh (\sqrt{\Omega} \eta)}{A \sinh (\sqrt{\Omega} \eta)+\mathrm{B}}\right)\right)^{-1}
\end{array}\right) \\
& u_{1_{7}}(x, t)=\left(\begin{array}{l}
\mp p \frac{\sqrt{6 k}}{\sqrt{\beta\left(4 q(v-1)-2-p^{2}\right)}} \pm 2 q \sqrt{\frac{6 k}{\beta\left(4 q(v-1)-2-p^{2}\right)}} \\
\left(\frac{1}{2(v-1)}\left(-p+\frac{ \pm \sqrt{\Omega\left(A^{2}-B^{2}\right)}-A \sqrt{\Omega} \cosh (\sqrt{\Omega} \eta)}{A \sinh (\sqrt{\Omega} \eta)+\mathrm{B}}\right)\right)^{-1}
\end{array}\right)
\end{aligned}
$$

where $A$ and $B$ are two non-zero real constants and satisfies the condition $A^{2}-B^{2}>0$

$$
\begin{aligned}
& u_{1_{8}}(x, t)=\left(\begin{array}{l}
\mp p \frac{\sqrt{6 k}}{\sqrt{\beta\left(4 q(v-1)-2-p^{2}\right)}} \pm 2 q \sqrt{\frac{6 k}{\beta\left(4 q(v-1)-2-p^{2}\right)}} \\
\left(\frac{-2 q \sqrt{\Omega} \cosh (1 / 2 \sqrt{\Omega})}{\sqrt{\Omega} \sinh (\sqrt{\Omega})+p \cosh (1 / 2 \sqrt{\Omega})}\right)^{-1}
\end{array}\right) \\
& u_{1_{9}}(x, t)=\left(\begin{array}{l}
\mp p \frac{\sqrt{6 k}}{\sqrt{\beta\left(4 q(v-1)-2-p^{2}\right)}} \pm 2 q \sqrt{\frac{6 k}{\beta\left(4 q(v-1)-2-p^{2}\right)}} \\
\left(\frac{2 q \sqrt{\Omega} \sinh ((1 / 2) \sqrt{\Omega})}{\sqrt{\Omega} \cosh (\sqrt{\Omega})-p \sinh ((1 / 2) \sqrt{\Omega})}\right)^{-1}
\end{array}\right) \\
& u_{1_{10}}(x, t)=\left(\begin{array}{l}
\mp p \frac{\sqrt{6 k}}{\sqrt{\beta\left(4 q(v-1)-2-p^{2}\right)}} \pm 2 q \sqrt{\frac{6 k}{\beta\left(4 q(v-1)-2-p^{2}\right)}} \\
\left(\frac{-2 q \sqrt{\Omega} \cosh ((1 / 2) \sqrt{\Omega})}{\sqrt{\Omega} \sin (\sqrt{\Omega})+p \cosh ((1 / 2) \sqrt{\Omega}) \pm i \sqrt{\Omega}}\right)^{-1}
\end{array}\right) \\
& u_{1_{11}}(x, t)=\left(\begin{array}{l}
\mp p \frac{\sqrt{6 k}}{\sqrt{\beta\left(4 q(v-1)-2-p^{2}\right)}} \pm 2 q \sqrt{\frac{6 k}{\beta\left(4 q(v-1)-2-p^{2}\right)}} \\
\left(\frac{2 q \sqrt{\Omega} \sinh ((1 / 2) \sqrt{\Omega})}{\sqrt{\Omega} \cosh (\sqrt{\Omega})-p \sinh ((1 / 2) \sqrt{\Omega}) \pm \sqrt{\Omega}}\right)^{-1}
\end{array}\right)
\end{aligned}
$$

when $\Omega=p^{2} ? 4 q(v-1)<0$ and $p(v-1) \neq 0($ or $q(v-1) \neq 0)$

$$
u_{1_{12}}(x, t)=\left(\begin{array}{l}
\mp p \frac{\sqrt{6 k}}{\sqrt{\beta\left(4 q(v-1)-2-p^{2}\right)}} \pm 2 q \sqrt{\frac{6 k}{\beta\left(4 q(v-1)-2-p^{2}\right)}} \\
\left(\frac{1}{2(v-1)}\left(-p+\sqrt{-\Omega} \tan \left(\frac{1}{2} \sqrt{-\Omega} \eta\right)\right)\right)^{-1}
\end{array}\right)
$$




$$
\begin{aligned}
& u_{1_{13}}(x, t)=\left(\begin{array}{l}
\mp p \frac{\sqrt{6 k}}{\sqrt{\beta\left(4 q(v-1)-2-p^{2}\right)}} \pm 2 q \sqrt{\frac{6 k}{\beta\left(4 q(v-1)-2-p^{2}\right)}} \\
\left(\frac{1}{2(v-1)}\left(-p+\sqrt{-\Omega} \cot \left(\frac{1}{2} \sqrt{-\Omega} \eta\right)\right)\right)^{-1}
\end{array}\right) \\
& u_{1_{14}}(x, t)=\left(\begin{array}{l}
\mp p \frac{\sqrt{6 k}}{\sqrt{\beta\left(4 q(v-1)-2-p^{2}\right)}} \pm 2 q \sqrt{\frac{6 k}{\beta\left(4 q(v-1)-2-p^{2}\right)}} \\
\left(\frac{1}{2(v-1)}\left(-\lambda+\sqrt{-\Omega}\left(\tan \left(\frac{1}{2} \sqrt{-\Omega} \eta\right) \pm \sec \left(\frac{1}{2} \sqrt{-\Omega} \eta\right)\right)\right)\right)^{-1}
\end{array}\right) \\
& u_{15}(x, t)=\left(\begin{array}{l}
\mp p \frac{\sqrt{6 k}}{\sqrt{\beta\left(4 q(v-1)-2-p^{2}\right)}} \pm 2 q \sqrt{\frac{6 k}{\beta\left(4 q(v-1)-2-p^{2}\right)}} \\
\left(-\frac{1}{2(v-1)}\left(p+\sqrt{-\Omega}\left(\cot \left(\frac{1}{2} \sqrt{-\Omega} \eta\right) \pm \csc \left(\frac{1}{2} \sqrt{-\Omega} \eta\right)\right)\right)\right)^{-1}
\end{array}\right) \\
& u_{1_{16}}(x, t)=\left(\begin{array}{l}
\mp p \frac{\sqrt{6 k}}{\sqrt{\beta\left(4 q(v-1)-2-p^{2}\right)}} \pm 2 q \sqrt{\frac{6 k}{\beta\left(4 q(v-1)-2-p^{2}\right)}} \\
\left(\frac{1}{4(v-1)}\left(-2 p+\sqrt{-\Omega}\left(\tan \left(\frac{1}{4} \sqrt{-\Omega} \eta\right)+\cot \left(\frac{1}{4} \sqrt{-\Omega} \eta\right)\right)\right)\right)^{-1}
\end{array}\right) \\
& u_{1,7}(x, t)=\left(\begin{array}{l}
\mp p \frac{\sqrt{6 k}}{\sqrt{\beta\left(4 q(v-1)-2-p^{2}\right)}} \pm 2 q \sqrt{\frac{6 k}{\beta\left(4 q(v-1)-2-p^{2}\right)}} \\
\left(\frac{1}{2(v-1)}\left(-p+\frac{ \pm \sqrt{-\Omega\left(A^{2}+B^{2}\right)}-A \sqrt{-\Omega} \cos (\sqrt{-\Omega} \eta)}{A \sin (\sqrt{-\Omega} \eta)+\mathrm{B}}\right)\right)^{-1}
\end{array}\right) \\
& u_{1_{18}}(x, t)=\left(\begin{array}{l}
\mp p \frac{\sqrt{6 k}}{\sqrt{\beta\left(4 q(v-1)-2-p^{2}\right)}} \pm 2 q \sqrt{\frac{6 k}{\beta\left(4 q(v-1)-2-p^{2}\right)}} \\
\left(\frac{1}{2(v-1)}\left(-p+\frac{ \pm \sqrt{-\Omega\left(A^{2}+B^{2}\right)}+A \sqrt{\Omega} \cos (\sqrt{-\Omega} \eta)}{A \sin (\sqrt{-\Omega} \eta)+\mathrm{B}}\right)\right)^{-1}
\end{array}\right)
\end{aligned}
$$

where $A$ and $B$ are two non-zero real constants

$$
u_{19}(x, t)=\left(\begin{array}{l}
\mp p \frac{\sqrt{6 k}}{\sqrt{\beta\left(4 q(v-1)-2-p^{2}\right)}} \pm 2 q \sqrt{\frac{6 k}{\beta\left(4 q(v-1)-2-p^{2}\right)}} \\
\left(\frac{-2 q \sqrt{-\Omega} \cos (1 / 2 \sqrt{-\Omega})}{\sqrt{-\Omega} \sin (\sqrt{-\Omega})+p \cos (1 / 2 \sqrt{-\Omega})}\right)^{-1}
\end{array}\right)
$$




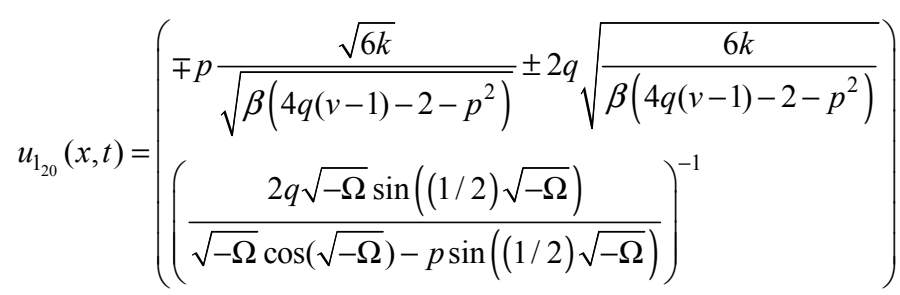

$$
\begin{aligned}
& u_{1_{21}}(x, t)=\left(\begin{array}{l}
\mp p \frac{\sqrt{6 k}}{\sqrt{\beta\left(4 q(v-1)-2-p^{2}\right)}} \pm 2 q \sqrt{\frac{6 k}{\beta\left(4 q(v-1)-2-p^{2}\right)}} \\
\left(\begin{array}{l}
-2 q \sqrt{-\Omega} \cos ((1 / 2) \sqrt{-\Omega}) \\
\sqrt{-\Omega} \sin (\sqrt{-\Omega})+p \cos ((1 / 2) \sqrt{-\Omega}) \pm \sqrt{-\Omega}
\end{array}\right)^{-1}
\end{array}\right) \\
& u_{1_{22}}(x, t)=\left(\begin{array}{l}
\mp p \frac{\sqrt{6 k}}{\sqrt{\beta\left(4 q(v-1)-2-p^{2}\right)}} \pm 2 q \sqrt{\frac{6 k}{\beta\left(4 q(v-1)-2-p^{2}\right)}} \\
\left(\frac{2 q \sqrt{-\Omega} \sin ((1 / 2) \sqrt{-\Omega})}{\sqrt{-\Omega} \cos (\sqrt{-\Omega})-p \sin ((1 / 2) \sqrt{-\Omega}) \pm \sqrt{-\Omega}}\right)^{-1}
\end{array}\right)
\end{aligned}
$$

when $\mu=0$ and $\lambda(v-1) \neq 0$

$$
\begin{aligned}
& u_{1_{23}}(x, t)=\left(\begin{array}{l}
\mp p \frac{\sqrt{6 k}}{\sqrt{\beta\left(4 q(v-1)-2-p^{2}\right)}} \pm 2 q \sqrt{\frac{6 k}{\beta\left(4 q(v-1)-2-p^{2}\right)}} \\
\left(-\frac{p k}{(v-1)(k+\cosh (p \eta)-p \sinh (p \eta))}\right)^{-1}
\end{array}\right) \\
& u_{1_{24}}(x, t)=\left(\begin{array}{l}
\mp p \frac{\sqrt{6 k}}{\sqrt{\beta\left(4 q(v-1)-2-p^{2}\right)}} \pm 2 q \sqrt{\frac{6 k}{\beta\left(4 q(v-1)-2-p^{2}\right)}} \\
\left(-\frac{\cosh (p \eta)+p \sinh (p \eta)}{(v-1)(k+\cosh (p \eta)+p \sinh (p \eta))}\right)^{-1}
\end{array}\right)
\end{aligned}
$$

where $\mathrm{k}$ is an arbitrary constant.

when $(v-1) \neq 0$ and $q=p=0$

$$
u_{\mathrm{l}_{25}}(x, t)=\left(\begin{array}{l}
\mp p \frac{\sqrt{6 k}}{\sqrt{\beta\left(4 q(v-1)-2-p^{2}\right)}} \pm 2 q \sqrt{\frac{6 k}{\beta\left(4 q(v-1)-2-p^{2}\right)}} \\
\left(-\frac{1}{(v-1) \eta+1}\right)^{-1}
\end{array}\right)
$$

where $\omega=\frac{4 k}{p^{2}-4 q(v-1)+2}, \eta=k x-\omega t, k$ is constant and $\mathrm{i}^{2}=-1$ in (17)-(41)

Substituting the solutions of the (6) (see appendix I) into (15) and simplifying we obtain the following solutions of our target equation (8)

when $\Omega=p^{2} ? 4 q(v-1)>0$ and $p(v-1) \neq 0($ or $q(v-1) \neq 0)$ 


$$
\begin{aligned}
& u_{2_{1}}(x, t)=\left(\begin{array}{l}
\mp p \frac{\sqrt{6 k}}{\sqrt{\beta\left(4 q(v-1)-2+p^{2}\right)}} \mp \frac{2 \sqrt{6 k}(v-1)}{\sqrt{\beta\left(4 q(v-1)-2+p^{2}\right)}} \\
\left(-\frac{1}{2(v-1)}\left(p+\sqrt{\Omega} \tanh \left(\frac{1}{2} \sqrt{\Omega} \eta\right)\right)\right)^{-1}
\end{array}\right) \\
& u_{2_{2}}(x, t)=\left(\begin{array}{l}
\mp p \frac{\sqrt{6 k}}{\sqrt{\beta\left(4 q(v-1)-2+p^{2}\right)}} \mp \frac{2 \sqrt{6 k}(v-1)}{\sqrt{\beta\left(4 q(v-1)-2+p^{2}\right)}} \\
\left(-\frac{1}{2(v-1)}\left(p+\sqrt{\Omega} \operatorname{coth}\left(\frac{1}{2} \sqrt{\Omega} \eta\right)\right)\right)^{-1}
\end{array}\right) \\
& u_{2_{3}}(x, t)=\left(\begin{array}{l}
\mp p \frac{\sqrt{6 k}}{\sqrt{\beta\left(4 q(v-1)-2+p^{2}\right)}} \mp \frac{2 \sqrt{6 k}(v-1)}{\sqrt{\beta\left(4 q(v-1)-2+p^{2}\right)}} \\
\left(-\frac{1}{2(v-1)}\left(p+\sqrt{\Omega}\left(\tanh \left(\frac{1}{2} \sqrt{\Omega} \eta\right) \pm i \sec h\left(\frac{1}{2} \sqrt{\Omega} \eta\right)\right)\right)\right)^{-1}
\end{array}\right) \\
& u_{2_{4}}(x, t)=\left(\begin{array}{l}
\mp p \frac{\sqrt{6 k}}{\sqrt{\beta\left(4 q(v-1)-2+p^{2}\right)}} \mp \frac{2 \sqrt{6 k}(v-1)}{\sqrt{\beta\left(4 q(v-1)-2+p^{2}\right)}} \\
\left(-\frac{1}{2(v-1)}\left(p+\sqrt{\Omega}\left(\operatorname{coth}\left(\frac{1}{2} \sqrt{\Omega} \eta\right) \pm \operatorname{csch}\left(\frac{1}{2} \sqrt{\Omega} \eta\right)\right)\right)\right)^{-1}
\end{array}\right) \\
& u_{2_{5}}(x, t)=\left(\begin{array}{l}
\mp p \frac{\sqrt{6 k}}{\sqrt{\beta\left(4 q(v-1)-2+p^{2}\right)}} \mp \frac{2 \sqrt{6 k}(v-1)}{\sqrt{\beta\left(4 q(v-1)-2+p^{2}\right)}} \\
\left(-\frac{1}{4(v-1)}\left(2 p+\sqrt{\Omega}\left(\tanh \left(\frac{1}{4} \sqrt{\Omega} \eta\right)+\operatorname{coth}\left(\frac{1}{4} \sqrt{\Omega} \eta\right)\right)\right)\right)^{-1}
\end{array}\right) \\
& u_{2_{6}}(x, t)=\left(\begin{array}{l}
\mp p \frac{\sqrt{6 k}}{\sqrt{\beta\left(4 q(v-1)-2+p^{2}\right)}} \mp \frac{2 \sqrt{6 k}(v-1)}{\sqrt{\beta\left(4 q(v-1)-2+p^{2}\right)}} \\
\left(\frac{1}{2(v-1)}\left(-p+\frac{ \pm \sqrt{\Omega\left(A^{2}-B^{2}\right)}-A \sqrt{\Omega} \cosh (\sqrt{\Omega} \eta)}{A \sinh (\sqrt{\Omega} \eta)+\mathrm{B}}\right)\right)^{-1}
\end{array}\right) \\
& u_{2_{7}}(x, t)=\left(\begin{array}{l}
\mp p \frac{\sqrt{6 k}}{\sqrt{\beta\left(4 q(v-1)-2+p^{2}\right)}} \mp \frac{2 \sqrt{6 k}(v-1)}{\sqrt{\beta\left(4 q(v-1)-2+p^{2}\right)}} \\
\left(\frac{1}{2(v-1)}\left(-p+\frac{ \pm \sqrt{\Omega\left(A^{2}-B^{2}\right)}-A \sqrt{\Omega} \cosh (\sqrt{\Omega} \eta)}{A \sinh (\sqrt{\Omega} \eta)+\mathrm{B}}\right)\right)^{-1}
\end{array}\right)
\end{aligned}
$$

where $A$ and $B$ are two non-zero real constants and satisfies the condition $A^{2}-B^{2}>0$

$$
u_{2_{8}}(x, t)=\left(\begin{array}{l}
\mp p \frac{\sqrt{6 k}}{\sqrt{\beta\left(4 q(v-1)-2+p^{2}\right)}} \mp \frac{2 \sqrt{6 k}(v-1)}{\sqrt{\beta\left(4 q(v-1)-2+p^{2}\right)}} \\
\left(\frac{-2 q \sqrt{\Omega} \cosh (1 / 2 \sqrt{\Omega})}{\sqrt{\Omega} \sinh (\sqrt{\Omega})+p \cosh (1 / 2 \sqrt{\Omega})}\right)^{-1}
\end{array}\right)
$$




$$
\begin{aligned}
& u_{2_{9}}(x, t)=\left(\begin{array}{l}
\mp p \frac{\sqrt{6 k}}{\sqrt{\beta\left(4 q(v-1)-2+p^{2}\right)}} \mp \frac{2 \sqrt{6 k}(v-1)}{\sqrt{\beta\left(4 q(v-1)-2+p^{2}\right)}} \\
\left(\frac{2 q \sqrt{\Omega} \sinh ((1 / 2) \sqrt{\Omega})}{\sqrt{\Omega} \cosh (\sqrt{\Omega})-p \sinh ((1 / 2) \sqrt{\Omega})}\right)^{-1}
\end{array}\right) \\
& u_{2_{10}}(x, t)=\left(\begin{array}{l}
\mp p \frac{\sqrt{6 k}}{\sqrt{\beta\left(4 q(v-1)-2+p^{2}\right)}} \mp \frac{2 \sqrt{6 k}(v-1)}{\sqrt{\beta\left(4 q(v-1)-2+p^{2}\right)}} \\
\left(\begin{array}{l}
-2 q \sqrt{\Omega} \cosh ((1 / 2) \sqrt{\Omega}) \\
\sqrt{\Omega} \sin (\sqrt{\Omega})+p \cosh ((1 / 2) \sqrt{\Omega}) \pm i \sqrt{\Omega}
\end{array}\right)^{-1}
\end{array}\right) \\
& u_{2_{11}}(x, t)=\left(\begin{array}{l}
\mp p \frac{\sqrt{6 k}}{\sqrt{\beta\left(4 q(v-1)-2+p^{2}\right)}} \mp \frac{2 \sqrt{6 k}(v-1)}{\sqrt{\beta\left(4 q(v-1)-2+p^{2}\right)}} \\
\left(\begin{array}{c}
2 q \sqrt{\Omega} \sinh ((1 / 2) \sqrt{\Omega}) \\
\sqrt{\Omega} \cosh (\sqrt{\Omega})-p \sinh ((1 / 2) \sqrt{\Omega}) \pm \sqrt{\Omega}
\end{array}\right)^{-1}
\end{array}\right)
\end{aligned}
$$

when $\Omega=p^{2} ? 4 q(v-1)<0$ and $p(v-1) \neq 0($ or $q(v-1) \neq 0)$

$$
\begin{gathered}
u_{2_{12}}(x, t)=\left(\begin{array}{l}
\mp p \frac{\sqrt{6 k}}{\sqrt{\beta\left(4 q(v-1)-2+p^{2}\right)}} \mp \frac{2 \sqrt{6 k}(v-1)}{\sqrt{\beta\left(4 q(v-1)-2+p^{2}\right)}} \\
\left(\frac{1}{2(v-1)}\left(-p+\sqrt{-\Omega} \tan \left(\frac{1}{2} \sqrt{-\Omega} \eta\right)\right)\right)^{-1}
\end{array}\right) \\
u_{2_{13}}(x, t)=\left(\begin{array}{l}
\left.\mp p \frac{\sqrt{6 k}}{\sqrt{\beta\left(4 q(v-1)-2+p^{2}\right)}} \mp \frac{2 \sqrt{6 k}(v-1)}{\sqrt{\beta\left(4 q(v-1)-2+p^{2}\right)}}\right) \\
\left(\frac{1}{2(v-1)}\left(-p+\sqrt{-\Omega} \cot \left(\frac{1}{2} \sqrt{-\Omega} \eta\right)\right)\right)^{-1}
\end{array}\right) \\
u_{2_{14}}(x, t)=\left(\begin{array}{l}
\mp p \frac{2 \sqrt{6 k}(v-1)}{\sqrt{\beta\left(4 q(v-1)-2+p^{2}\right)}} \mp \frac{\sqrt{6 k}}{\sqrt{\beta\left(4 q(v-1)-2+p^{2}\right)}} \\
\left(\frac{1}{2(v-1)}\left(-\lambda+\sqrt{-\Omega}\left(\tan \left(\frac{1}{2} \sqrt{-\Omega} \eta\right) \pm \sec \left(\frac{1}{2} \sqrt{-\Omega} \eta\right)\right)\right)\right)^{-1}
\end{array}\right) \\
u_{2_{15}}(x, t)=\left(\begin{array}{l}
\left.\mp p \frac{2 \sqrt{6 k}(v-1)}{\sqrt{\beta\left(4 q(v-1)-2+p^{2}\right)}} \mp \frac{1}{\sqrt{\beta\left(4 q(v-1)-2+p^{2}\right)}}\left(p+\sqrt{-\Omega}\left(\cot \left(\frac{1}{2} \sqrt{-\Omega} \eta\right) \pm \csc \left(\frac{1}{2} \sqrt{-\Omega} \eta\right)\right)\right)\right)^{-1}
\end{array}\right) \\
\left(-\frac{1}{2(v-1)}(p)\right.
\end{gathered}
$$




$$
\begin{aligned}
& u_{2_{16}}(x, t)=\left(\begin{array}{l}
\mp p \frac{\sqrt{6 k}}{\sqrt{\beta\left(4 q(v-1)-2+p^{2}\right)}} \mp \frac{2 \sqrt{6 k}(v-1)}{\sqrt{\beta\left(4 q(v-1)-2+p^{2}\right)}} \\
\left(\frac{1}{4(v-1)}\left(-2 p+\sqrt{-\Omega}\left(\tan \left(\frac{1}{4} \sqrt{-\Omega} \eta\right)+\cot \left(\frac{1}{4} \sqrt{-\Omega} \eta\right)\right)\right)\right)^{-1}
\end{array}\right) \\
& u_{2_{17}}(x, t)=\left(\begin{array}{l}
\mp p \frac{\sqrt{6 k}}{\sqrt{\beta\left(4 q(v-1)-2+p^{2}\right)}} \mp \frac{2 \sqrt{6 k}(v-1)}{\sqrt{\beta\left(4 q(v-1)-2+p^{2}\right)}} \\
\left(\frac{1}{2(v-1)}\left(-p+\frac{ \pm \sqrt{-\Omega\left(A^{2}+B^{2}\right)}-A \sqrt{-\Omega} \cos (\sqrt{-\Omega} \eta)}{A \sin (\sqrt{-\Omega} \eta)+\mathrm{B}}\right)\right)^{-1}
\end{array}\right) \\
& u_{2_{18}}(x, t)=\left(\begin{array}{l}
\mp p \frac{2 \sqrt{6 k}(v-1)}{\sqrt{\beta\left(4 q(v-1)-2+p^{2}\right)}} \mp \frac{\sqrt{6 k}}{\sqrt{\beta\left(4 q(v-1)-2+p^{2}\right)}} \\
\left(\frac{1}{2(v-1)}\left(-p+\frac{ \pm \sqrt{-\Omega\left(A^{2}+B^{2}\right)}+A \sqrt{\Omega} \cos (\sqrt{-\Omega} \eta)}{A \sin (\sqrt{-\Omega} \eta)+\mathrm{B}}\right)\right)
\end{array}\right)
\end{aligned}
$$

where $A$ and $B$ are two non-zero real constants

$$
\begin{aligned}
& u_{2_{19}}(x, t)=\left(\begin{array}{l}
\mp p \frac{\sqrt{6 k}}{\sqrt{\beta\left(4 q(v-1)-2+p^{2}\right)}} \mp \frac{2 \sqrt{6 k}(v-1)}{\sqrt{\beta\left(4 q(v-1)-2+p^{2}\right)}} \\
\left(\frac{-2 q \sqrt{-\Omega} \cos (1 / 2 \sqrt{-\Omega})}{\sqrt{-\Omega} \sin (\sqrt{-\Omega})+p \cos (1 / 2 \sqrt{-\Omega})}\right)^{-1}
\end{array}\right) \\
& u_{2_{20}}(x, t)=\left(\begin{array}{l}
\mp p \frac{\sqrt{6 k}}{\sqrt{\beta\left(4 q(v-1)-2+p^{2}\right)}} \mp \frac{2 \sqrt{6 k}(v-1)}{\sqrt{\beta\left(4 q(v-1)-2+p^{2}\right)}} \\
\left(\frac{2 q \sqrt{-\Omega} \sin ((1 / 2) \sqrt{-\Omega})}{\sqrt{-\Omega} \cos (\sqrt{-\Omega})-p \sin ((1 / 2) \sqrt{-\Omega})}\right)^{-1}
\end{array}\right) \\
& u_{21}(x, t)=\left(\begin{array}{l}
\mp p \frac{\sqrt{6 k}}{\sqrt{\beta\left(4 q(v-1)-2+p^{2}\right)}} \mp \frac{2 \sqrt{6 k}(v-1)}{\sqrt{\beta\left(4 q(v-1)-2+p^{2}\right)}} \\
\left(\frac{-2 q \sqrt{-\Omega} \cos ((1 / 2) \sqrt{-\Omega})}{\sqrt{-\Omega} \sin (\sqrt{-\Omega})+p \cos ((1 / 2) \sqrt{-\Omega}) \pm \sqrt{-\Omega}}\right)^{-1}
\end{array}\right) \\
& u_{22}(x, t)=\left(\begin{array}{l}
\mp p \frac{\sqrt{6 k}}{\sqrt{\beta\left(4 q(v-1)-2+p^{2}\right)}} \mp \frac{2 \sqrt{6 k}(v-1)}{\sqrt{\beta\left(4 q(v-1)-2+p^{2}\right)}} \\
\left(\begin{array}{c}
2 q \sqrt{-\Omega} \sin ((1 / 2) \sqrt{-\Omega}) \\
\sqrt{-\Omega} \cos (\sqrt{-\Omega})-p \sin ((1 / 2) \sqrt{-\Omega}) \pm \sqrt{-\Omega}
\end{array}\right)^{-1}
\end{array}\right)
\end{aligned}
$$

when $\mu=0$ and $\lambda(v-1) \neq 0$ 


$$
\begin{aligned}
& u_{2_{23}}(x, t)=\left(\begin{array}{l}
\mp p \frac{\sqrt{6 k}}{\sqrt{\beta\left(4 q(v-1)-2+p^{2}\right)}} \mp \frac{2 \sqrt{6 k}(v-1)}{\sqrt{\beta\left(4 q(v-1)-2+p^{2}\right)}} \\
\left(-\frac{p k}{(v-1)(k+\cosh (p \eta)-p \sinh (p \eta))}\right)^{-1}
\end{array}\right) \\
& u_{2_{24}}(x, t)=\left(\begin{array}{l}
\mp p \frac{\sqrt{6 k}}{\sqrt{\beta\left(4 q(v-1)-2+p^{2}\right)}} \mp \frac{2 \sqrt{6 k}(v-1)}{\sqrt{\beta\left(4 q(v-1)-2+p^{2}\right)}} \\
\left(-\frac{\cosh (p \eta)+p \sinh (p \eta)}{(v-1)(k+\cosh (p \eta)+p \sinh (p \eta))}\right)^{-1}
\end{array}\right)
\end{aligned}
$$

where $\mathrm{k}$ is an arbitrary constant.

when $(v-1) \neq 0$ and $q=p=0$

$$
u_{25}(x, t)=\left(\begin{array}{l}
\mp p \frac{\sqrt{6 k}}{\sqrt{\beta\left(4 q(v-1)-2+p^{2}\right)}} \mp \frac{2 \sqrt{6 k}(v-1)}{\sqrt{\beta\left(4 q(v-1)-2+p^{2}\right)}} \\
\left(-\frac{1}{(v-1) \eta+1}\right)^{-1}
\end{array}\right)
$$

where $\omega=\frac{-4 k}{p^{2}-4 q(v-1)-2}, \eta=k x-\omega t, k$ is constant and $\mathrm{i}^{2}=-1$ in (45)-(66)

Substituting the solutions of the (6) (see appendix I) into (16) and simplifying we obtain the following solutions of our target equation (8). For simplicity we do not plug in the coefficients in (13) $\alpha_{0}, \alpha_{1}$, and $\beta_{1}$ in following solutions.

when $\Omega=p^{2} ? 4 q(v-1)>0$ and $p(v-1) \neq 0($ or $q(v-1) \neq 0)$

$$
\begin{aligned}
& u_{3_{1}}(x, t)=\left(\begin{array}{l}
\alpha_{0}+\alpha_{1}\left(-\frac{1}{2(v-1)}\left(p+\sqrt{\Omega} \tanh \left(\frac{1}{2} \sqrt{\Omega} \eta\right)\right)\right)+ \\
\beta_{1}\left(-\frac{1}{2(v-1)}\left(p+\sqrt{\Omega} \tanh \left(\frac{1}{2} \sqrt{\Omega} \eta\right)\right)\right)^{-1}
\end{array}\right) \\
& u_{3_{2}}(x, t)=\left(\begin{array}{l}
\left.\alpha_{0}+\alpha_{1}\left(-\frac{1}{2(v-1)}\left(p+\sqrt{\Omega} \operatorname{coth}\left(\frac{1}{2} \sqrt{\Omega} \eta\right)\right)\right)+\right) \\
\beta_{1}\left(-\frac{1}{2(v-1)}\left(p+\sqrt{\Omega} \operatorname{coth}\left(\frac{1}{2} \sqrt{\Omega} \eta\right)\right)\right)^{-1}
\end{array}\right) \\
& u_{3_{3}}(x, t)=\left(\begin{array}{l}
\alpha_{0}+\alpha_{1}\left(-\frac{1}{2(v-1)}\left(p+\sqrt{\Omega}\left(\tanh \left(\frac{1}{2} \sqrt{\Omega} \eta\right) \pm i \sec h\left(\frac{1}{2} \sqrt{\Omega} \eta\right)\right)\right)\right)+ \\
\beta_{1}\left(-\frac{1}{2(v-1)}\left(p+\sqrt{\Omega}\left(\tanh \left(\frac{1}{2} \sqrt{\Omega} \eta\right) \pm i \sec h\left(\frac{1}{2} \sqrt{\Omega} \eta\right)\right)\right)\right)^{-1}
\end{array}\right) \\
& u_{3_{4}}(x, t)=\left(\begin{array}{l}
\alpha_{0}+\alpha_{1}\left(-\frac{1}{2(v-1)}\left(p+\sqrt{\Omega}\left(\operatorname{coth}\left(\frac{1}{2} \sqrt{\Omega} \eta\right) \pm \csc h\left(\frac{1}{2} \sqrt{\Omega} \eta\right)\right)\right)\right)+ \\
\beta_{1}\left(-\frac{1}{2(v-1)}\left(p+\sqrt{\Omega}\left(\operatorname{coth}\left(\frac{1}{2} \sqrt{\Omega} \eta\right) \pm \csc h\left(\frac{1}{2} \sqrt{\Omega} \eta\right)\right)\right)\right)^{-1}
\end{array}\right)
\end{aligned}
$$




$$
\begin{gathered}
u_{3_{5}}(x, t)=\left(\begin{array}{l}
\alpha_{0}+\alpha_{1}\left(-\frac{1}{4(v-1)}\left(2 p+\sqrt{\Omega}\left(\tanh \left(\frac{1}{4} \sqrt{\Omega} \eta\right)+\operatorname{coth}\left(\frac{1}{4} \sqrt{\Omega} \eta\right)\right)\right)+\right. \\
\beta_{1}\left(-\frac{1}{4(v-1)}\left(2 p+\sqrt{\Omega}\left(\tanh \left(\frac{1}{4} \sqrt{\Omega} \eta\right)+\operatorname{coth}\left(\frac{1}{4} \sqrt{\Omega} \eta\right)\right)\right)\right)^{-1}
\end{array}\right) \\
u_{3_{6}}(x, t)=\left(\begin{array}{l}
\alpha_{0}+\alpha_{1}\left(\frac{1}{2(v-1)}\left(-p+\frac{ \pm \sqrt{\Omega\left(A^{2}-B^{2}\right)}-A \sqrt{\Omega} \cosh (\sqrt{\Omega} \eta)}{A \sinh (\sqrt{\Omega} \eta)+\mathrm{B}}\right)\right)+ \\
\beta_{1}\left(\frac{1}{2(v-1)}\left(-p+\frac{ \pm \sqrt{\Omega\left(A^{2}-B^{2}\right)}-A \sqrt{\Omega} \cosh (\sqrt{\Omega} \eta)}{A \sinh (\sqrt{\Omega} \eta)+\mathrm{B}}\right)\right)^{-1}
\end{array}\right) \\
u_{3_{7}}(x, t)=\left(\begin{array}{l}
\alpha_{0}+\alpha_{1}\left(\frac{1}{2(v-1)}\left(-p+\frac{ \pm \sqrt{\Omega\left(A^{2}-B^{2}\right)}-A \sqrt{\Omega} \cosh (\sqrt{\Omega} \eta)}{A \sinh (\sqrt{\Omega} \eta)+\mathrm{B}}\right)\right)+ \\
\beta_{1}\left(\frac{1}{2(v-1)}\left(-p+\frac{ \pm \sqrt{\Omega\left(A^{2}-B^{2}\right)}-A \sqrt{\Omega} \cosh (\sqrt{\Omega} \eta)}{A \sinh (\sqrt{\Omega} \eta)+\mathrm{B}}\right)\right)^{-1}
\end{array}\right)
\end{gathered}
$$

where $A$ and $B$ are two non-zero real constants and satisfies the condition $A^{2}-B^{2}>0$

$$
\begin{gathered}
u_{3_{8}}(x, t)=\left(\begin{array}{l}
\alpha_{0}+\alpha_{1}\left(\frac{-2 \mu \sqrt{\Omega} \cosh (1 / 2 \sqrt{\Omega})}{\sqrt{\Omega} \sinh (\sqrt{\Omega})+\lambda \cosh (1 / 2 \sqrt{\Omega})}\right)+ \\
\beta_{1}\left(\frac{-2 \mu \sqrt{\Omega} \cosh (1 / 2 \sqrt{\Omega})}{\sqrt{\Omega} \sinh (\sqrt{\Omega})+\lambda \cosh (1 / 2 \sqrt{\Omega})}\right)^{-1}
\end{array}\right) \\
u_{3_{9}}(x, t)=\left(\begin{array}{c}
\alpha_{0}+\alpha_{1}\left(\frac{2 \mu \sqrt{\Omega} \sinh ((1 / 2) \sqrt{\Omega})}{\sqrt{\Omega} \cosh (\sqrt{\Omega})-\lambda \sinh ((1 / 2) \sqrt{\Omega})}\right)+ \\
\beta_{1}\left(\frac{2 \mu \sqrt{\Omega} \sinh ((1 / 2) \sqrt{\Omega})}{\sqrt{\Omega} \cosh (\sqrt{\Omega})-\lambda \sinh ((1 / 2) \sqrt{\Omega})}\right)^{-1}
\end{array}\right) \\
u_{3_{10}}(x, t)=\left(\begin{array}{c}
-2 \mu \sqrt{\Omega} \cosh ((1 / 2) \sqrt{\Omega}) \\
\alpha_{0}+\alpha_{1}\left(\frac{-2 \mu \sqrt{\Omega} \cosh ((1 / 2) \sqrt{\Omega})}{\sqrt{\Omega} \sinh (\sqrt{\Omega})+\lambda \cosh ((1 / 2) \sqrt{\Omega}) \pm i \sqrt{\Omega}}\right)+ \\
\beta_{1}\left(\frac{-1}{\sqrt{\Omega} \sinh (\sqrt{\Omega})+\lambda \cosh ((1 / 2) \sqrt{\Omega}) \pm i \sqrt{\Omega}}\right)^{-1}
\end{array}\right) \\
u_{3_{11}}(x, t)=\left(\begin{array}{c}
2 \mu \sqrt{\Omega} \sinh ((1 / 2) \sqrt{\Omega}) \\
\alpha_{0}+\alpha_{1}\left(\frac{2 \mu \sqrt{\Omega} \sinh ((1 / 2) \sqrt{\Omega})}{\sqrt{\Omega} \cosh (\sqrt{\Omega})-\lambda \sinh ((1 / 2) \sqrt{\Omega}) \pm \sqrt{\Omega}}\right)+ \\
\beta_{1}\left(\frac{\cosh (\sqrt{\Omega})-\lambda \sinh ((1 / 2) \sqrt{\Omega}) \pm \sqrt{\Omega}}{\sqrt{\Omega}}\right)^{-1}
\end{array}\right)
\end{gathered}
$$

when $\Omega=\lambda^{2} ? 4 \mu(v-1)<0$ and $\lambda(v-1) \neq 0($ or $\mu(v-1) \neq 0)$ 


$$
\begin{aligned}
& u_{3_{12}}(x, t)=\left(\begin{array}{l}
\alpha_{0}+\alpha_{1}\left(\frac{1}{2(v-1)}\left(-\lambda+\sqrt{-\Omega} \tan \left(\frac{1}{2} \sqrt{-\Omega} \eta\right)\right)\right)+ \\
\beta_{1}\left(\frac{1}{2(v-1)}\left(-\lambda+\sqrt{-\Omega} \tan \left(\frac{1}{2} \sqrt{-\Omega} \eta\right)\right)\right)^{-1}
\end{array}\right) \\
& u_{3_{13}}(x, t)=\left(\begin{array}{l}
\alpha_{0}+\alpha_{1}\left(\frac{1}{2(v-1)}\left(-\lambda+\sqrt{-\Omega} \cot \left(\frac{1}{2} \sqrt{-\Omega} \eta\right)\right)\right)+ \\
\beta_{1}\left(\frac{1}{2(v-1)}\left(-\lambda+\sqrt{-\Omega} \cot \left(\frac{1}{2} \sqrt{-\Omega} \eta\right)\right)\right)^{-1}
\end{array}\right) \\
& u_{3_{14}} u(x, t)=\left(\begin{array}{l}
\alpha_{0}+\alpha_{1}\left(\frac{1}{2(v-1)}\left(-\lambda+\sqrt{-\Omega}\left(\tan \left(\frac{1}{2} \sqrt{-\Omega} \eta\right) \pm \sec \left(\frac{1}{2} \sqrt{-\Omega} \eta\right)\right)\right)\right)+ \\
\beta_{1}\left(\frac{1}{2(v-1)}\left(-\lambda+\sqrt{-\Omega}\left(\tan \left(\frac{1}{2} \sqrt{-\Omega} \eta\right) \pm \sec \left(\frac{1}{2} \sqrt{-\Omega} \eta\right)\right)\right)\right)^{-1}
\end{array}\right) \\
& u_{3_{15}}(x, t)=\left(\begin{array}{l}
\alpha_{0}+\alpha_{1}\left(-\frac{1}{2(v-1)}\left(\lambda+\sqrt{-\Omega}\left(\cot \left(\frac{1}{2} \sqrt{-\Omega} \eta\right) \pm \csc \left(\frac{1}{2} \sqrt{-\Omega} \eta\right)\right)\right)\right)+ \\
\beta_{1}\left(-\frac{1}{2(v-1)}\left(\lambda+\sqrt{-\Omega}\left(\cot \left(\frac{1}{2} \sqrt{-\Omega} \eta\right) \pm \csc \left(\frac{1}{2} \sqrt{-\Omega} \eta\right)\right)\right)\right)^{-1}
\end{array}\right) \\
& u_{3_{16}}(x, t)=\left(\begin{array}{l}
\alpha_{0}+\alpha_{1}\left(\frac{1}{4(v-1)}\left(-2 \lambda+\sqrt{-\Omega}\left(\tan \left(\frac{1}{4} \sqrt{-\Omega} \eta\right)+\cot \left(\frac{1}{4} \sqrt{-\Omega} \eta\right)\right)\right)\right)+ \\
\beta_{1}\left(\frac{1}{4(v-1)}\left(-2 \lambda+\sqrt{-\Omega}\left(\tan \left(\frac{1}{4} \sqrt{-\Omega} \eta\right)+\cot \left(\frac{1}{4} \sqrt{-\Omega} \eta\right)\right)\right)\right)^{-1}
\end{array}\right) \\
& u_{3_{17}}(x, t)=\left(\begin{array}{l}
\alpha_{0}+\alpha_{1}\left(\frac{1}{2(v-1)}\left(-\lambda+\frac{ \pm \sqrt{-\Omega\left(A^{2}+B^{2}\right)}-A \sqrt{-\Omega} \cos (\sqrt{-\Omega} \eta)}{A \sin (\sqrt{-\Omega} \eta)+\mathrm{B}}\right)\right)+ \\
\beta_{1}\left(\frac{1}{2(v-1)}\left(-\lambda+\frac{ \pm \sqrt{-\Omega\left(A^{2}+B^{2}\right)}-A \sqrt{-\Omega} \cos (\sqrt{-\Omega} \eta)}{A \sin (\sqrt{-\Omega} \eta)+\mathrm{B}}\right)\right)^{-1}
\end{array}\right) \\
& u_{3_{18}}(x, t)=\left(\begin{array}{l}
\alpha_{0}+\alpha_{1}\left(\frac{1}{2(v-1)}\left(-\lambda+\frac{ \pm \sqrt{-\Omega\left(A^{2}+B^{2}\right)}+A \sqrt{\Omega} \cos (\sqrt{-\Omega} \eta)}{A \sin (\sqrt{-\Omega} \eta)+\mathrm{B}}\right)\right)+ \\
\beta_{1}\left(\frac{1}{2(v-1)}\left(-\lambda+\frac{ \pm \sqrt{-\Omega\left(A^{2}+B^{2}\right)}+A \sqrt{\Omega} \cos (\sqrt{-\Omega} \eta)}{A \sin (\sqrt{-\Omega} \eta)+\mathrm{B}}\right)\right)^{-1}
\end{array}\right)
\end{aligned}
$$

where $A$ and $B$ are two non-zero real constants

$$
u_{3_{19}}(x, t)=\left(\begin{array}{l}
\alpha_{0}+\alpha_{1}\left(\frac{-2 \mu \sqrt{-\Omega} \cos (1 / 2 \sqrt{-\Omega})}{\sqrt{-\Omega} \sin (\sqrt{-\Omega})+\lambda \cos (1 / 2 \sqrt{-\Omega})}\right)+ \\
\beta_{1}\left(\frac{-2 \mu \sqrt{-\Omega} \cos (1 / 2 \sqrt{-\Omega})}{\sqrt{-\Omega} \sin (\sqrt{-\Omega})+\lambda \cos (1 / 2 \sqrt{-\Omega})}\right)^{-1}
\end{array}\right)
$$




$$
\begin{aligned}
& u_{3_{20}}(x, t)=\left(\begin{array}{l}
\alpha_{0}+\alpha_{1}\left(\frac{2 \mu \sqrt{-\Omega} \sin ((1 / 2) \sqrt{-\Omega})}{\sqrt{-\Omega} \cos (\sqrt{-\Omega})-\lambda \sin ((1 / 2) \sqrt{-\Omega})}\right)+ \\
\beta_{1}\left(\frac{2 \mu \sqrt{-\Omega} \sin ((1 / 2) \sqrt{-\Omega})}{\sqrt{-\Omega} \cos (\sqrt{-\Omega})-\lambda \sin ((1 / 2) \sqrt{-\Omega})}\right)^{-1}
\end{array}\right) \\
& u_{3_{21}}(x, t)=\left(\begin{array}{c}
\alpha_{0}+\alpha_{1}\left(\frac{-2 \mu \sqrt{-\Omega} \cos ((1 / 2) \sqrt{-\Omega})}{\sqrt{-\Omega} \sin (\sqrt{-\Omega})+\lambda \cos ((1 / 2) \sqrt{-\Omega}) \pm \sqrt{-\Omega}}\right)+ \\
\beta_{1}\left(\frac{-2 \mu \sqrt{-\Omega} \cos ((1 / 2) \sqrt{-\Omega})}{\sqrt{-\Omega} \sin (\sqrt{-\Omega})+\lambda \cos ((1 / 2) \sqrt{-\Omega}) \pm \sqrt{-\Omega}}\right)^{-1}
\end{array}\right) \\
& u_{3_{22}}(x, t)=\left(\begin{array}{l}
\alpha_{0}+\alpha_{1}\left(\frac{2 \mu \sqrt{-\Omega} \sin ((1 / 2) \sqrt{-\Omega})}{\sqrt{-\Omega} \cos (\sqrt{-\Omega})-\lambda \sin ((1 / 2) \sqrt{-\Omega}) \pm \sqrt{-\Omega}}\right)+ \\
\beta_{1}\left(\frac{2 \mu \sqrt{-\Omega} \sin ((1 / 2) \sqrt{-\Omega})}{\sqrt{-\Omega} \cos (\sqrt{-\Omega})-\lambda \sin ((1 / 2) \sqrt{-\Omega}) \pm \sqrt{-\Omega}}\right)^{-1}
\end{array}\right)
\end{aligned}
$$

when $\mu=0$ and $\lambda(v-1) \neq 0$

$$
\begin{aligned}
& u_{3_{23}}(x, t)=\left(\begin{array}{l}
\alpha_{0}+\alpha_{1}\left(-\frac{\lambda k}{(v-1)(k+\cosh (\lambda \eta)-\lambda \sinh (\lambda \eta))}\right)+ \\
\beta_{1}\left(-\frac{\lambda k}{(v-1)(k+\cosh (\lambda \eta)-\lambda \sinh (\lambda \eta))}\right)^{-1}
\end{array}\right) \\
& u_{3_{24}}(x, t)=\left(\begin{array}{l}
\alpha_{0}+\alpha_{1}\left(-\frac{\cosh (\lambda \eta)+\lambda \sinh (\lambda \eta)}{(v-1)(k+\cosh (\lambda \eta)+\lambda \sinh (\lambda \eta))}\right)+ \\
\beta_{1}\left(-\frac{\cosh (\lambda \eta)+\lambda \sinh (\lambda \eta)}{(v-1)(k+\cosh (\lambda \eta)+\lambda \sinh (\lambda \eta))}\right)^{-1}
\end{array}\right)
\end{aligned}
$$

where $k$ is an arbitrary constant.

when $(v-1) \neq 0$ and $q=p=0$

$$
u_{3_{25}}(x, t)=\left(\alpha_{0}+\alpha_{1}\left(-\frac{1}{(v-1) \eta+1}\right)+\beta_{1}\left(-\frac{1}{(v-1) \eta+1}\right)^{-1}\right)
$$

where $\omega=\frac{4 k}{p^{2}+8 q(v-1)+2}, \eta=k x-\omega t, k$ is constant and $\mathrm{i}^{2}=-1$ in (67)-(91)

\section{Results and Discussion}

Hassibun Naher et al. [29] applied the proposed method, improved $\left(G^{\prime} / G\right)$ - expansion method to target equation, that is, the Simplified Modified Camassa Holm (MCH) where
$G=G(\eta)$ satisfies $G^{\prime \prime}+\lambda G^{\prime}+\mu G=0$ and obtained some exact traveling wave solutions in terms of hyperbolic, periodic and rational functions. Another authors, Liu et al. [28] applied the basic $\left(G^{\prime} / G\right)$ - expansion method to the Simplified Modified Camassa Holm $(\mathrm{MCH})$ which is also our target equation where $G=G(\eta)$ satisfies $G^{\prime \prime}+\lambda G^{\prime}+\mu G=0$ and found a few exact traveling wave solutions as compared to [29] and our solutions. In our case, 
we applied the improved $\left(G^{\prime} / G\right)$ - expansion method to the Simplified Modified Camassa Holm (MCH) where $G=G(\eta) \quad$ satisfies $\quad G G^{\prime \prime}=p G G^{\prime}+q G^{2}+v\left(G^{\prime}\right)^{2}$

Consequently, we have constructed more general solutions and many new exact traveling solutions. These new exact traveling wave solutions are such as $u_{1_{3}}-u_{1_{11}}, u_{1_{14}}-u_{1_{25}}, u_{2_{3}}-u_{2_{11}}, u_{2_{14}}-u_{2_{25}}, u_{3_{3}}-u_{3_{11}}$, and $u_{3_{14}}-u_{3_{25}}$ of the solutions as follows; which are not being revealed in the previous literatures.
Furthermore, it is important to point out that some of our obtained solutions are in good agreement with the existing results which verifies our other solutions.

\section{Graphs of Some of the Solutions}

In order to substantiate we exemplified the graphs of some

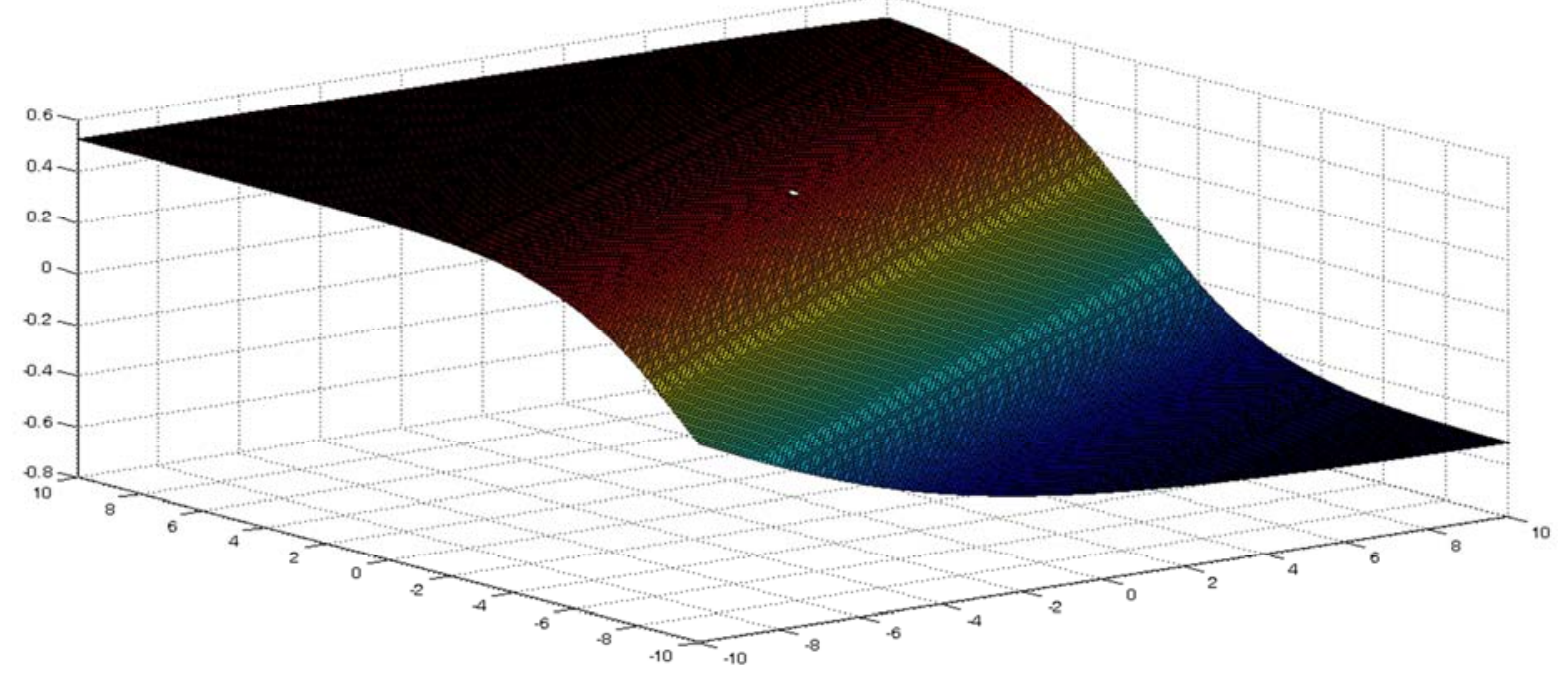

Figure 1. The graph of $u_{1_{4}}(x, t)$ for $k=-1, v=4, q=2, p=17 \& B=3$.

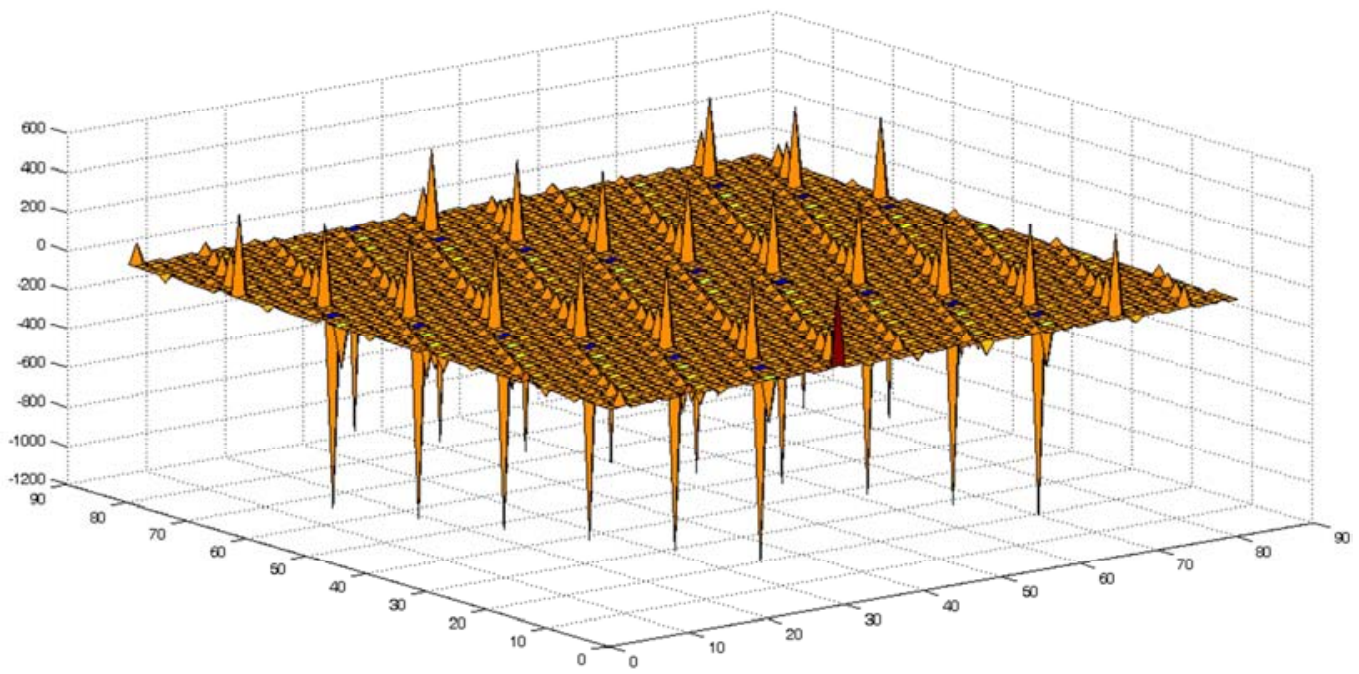

Figure 2. The graph of $u_{1_{13}}(x, t) \quad k=2, v-2, q=-1, p=1, B=2$. 


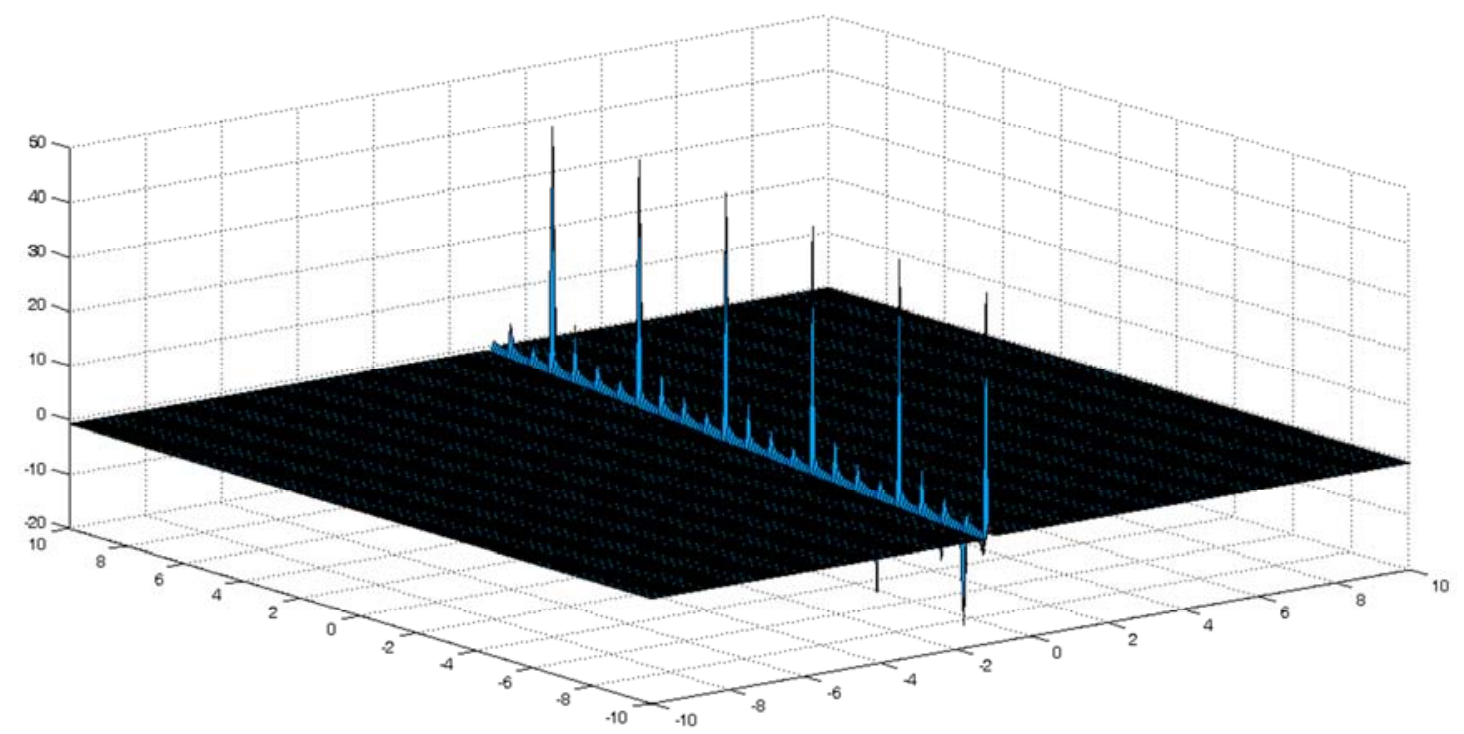

Figure 3. The graph of $u_{1_{6}}(x, t)$ for $k=2, v=-1, q=4, p=-1$.

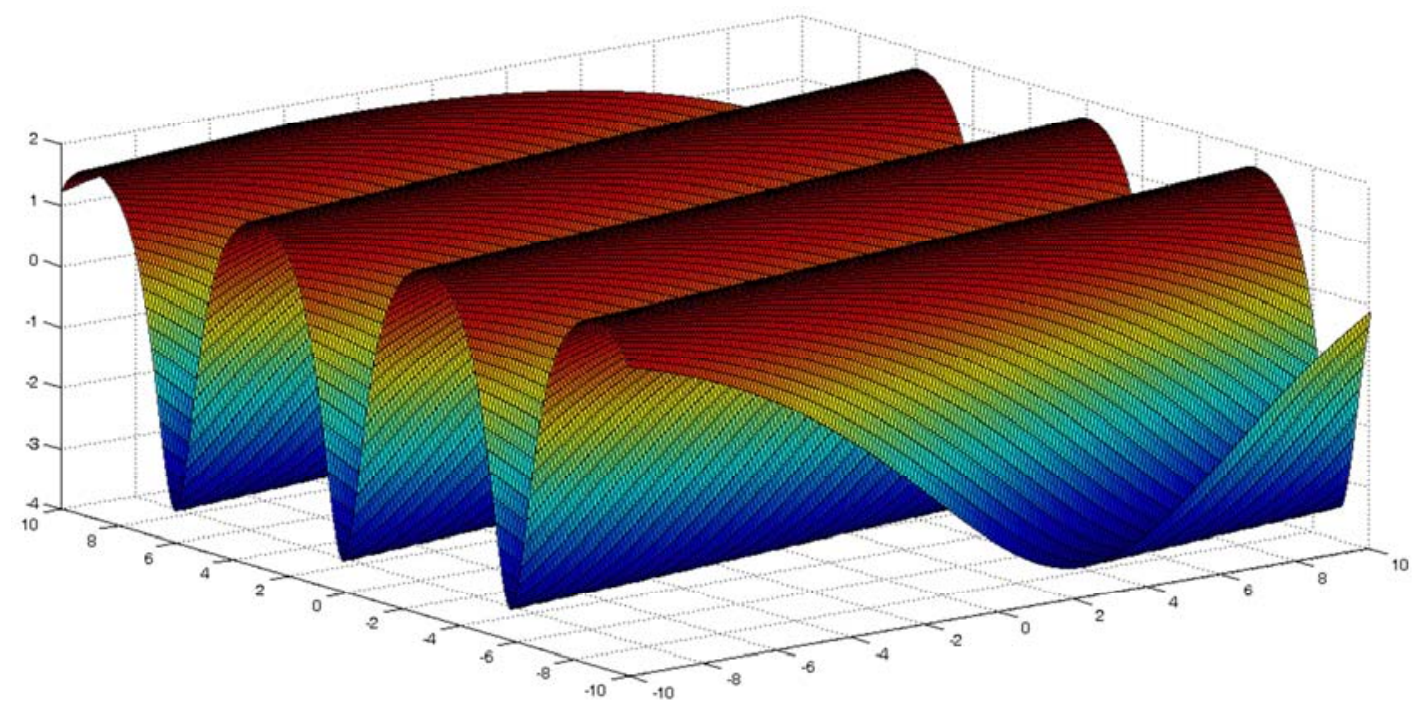

Figure 4. The graph of $u_{16}(x, t)$ for $k=-0.25, v=2, q=0.5, p=0.75, A=4 \& B=3 ;$ (Oscillating Surface).

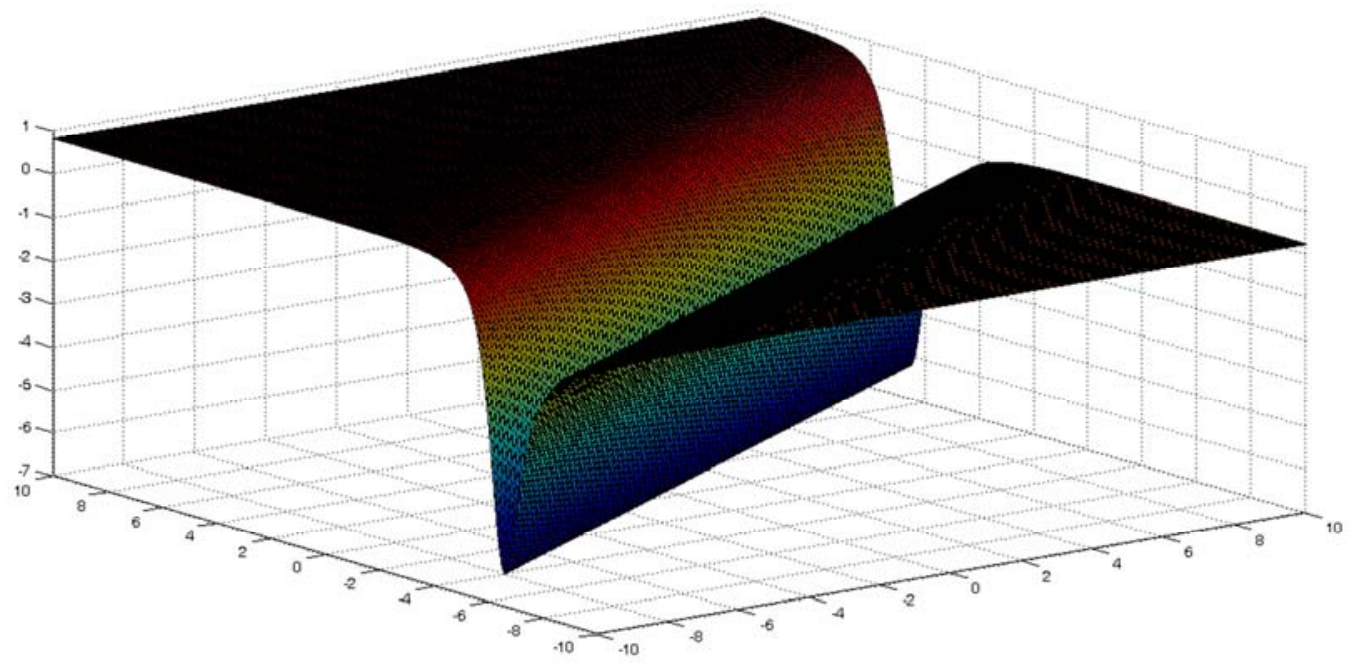

Figure 5. The Graph of $u_{2_{3}}(x, t)$ for $k=-2, v=2, q=1 / 12, p=0.75 \& B=2$. 


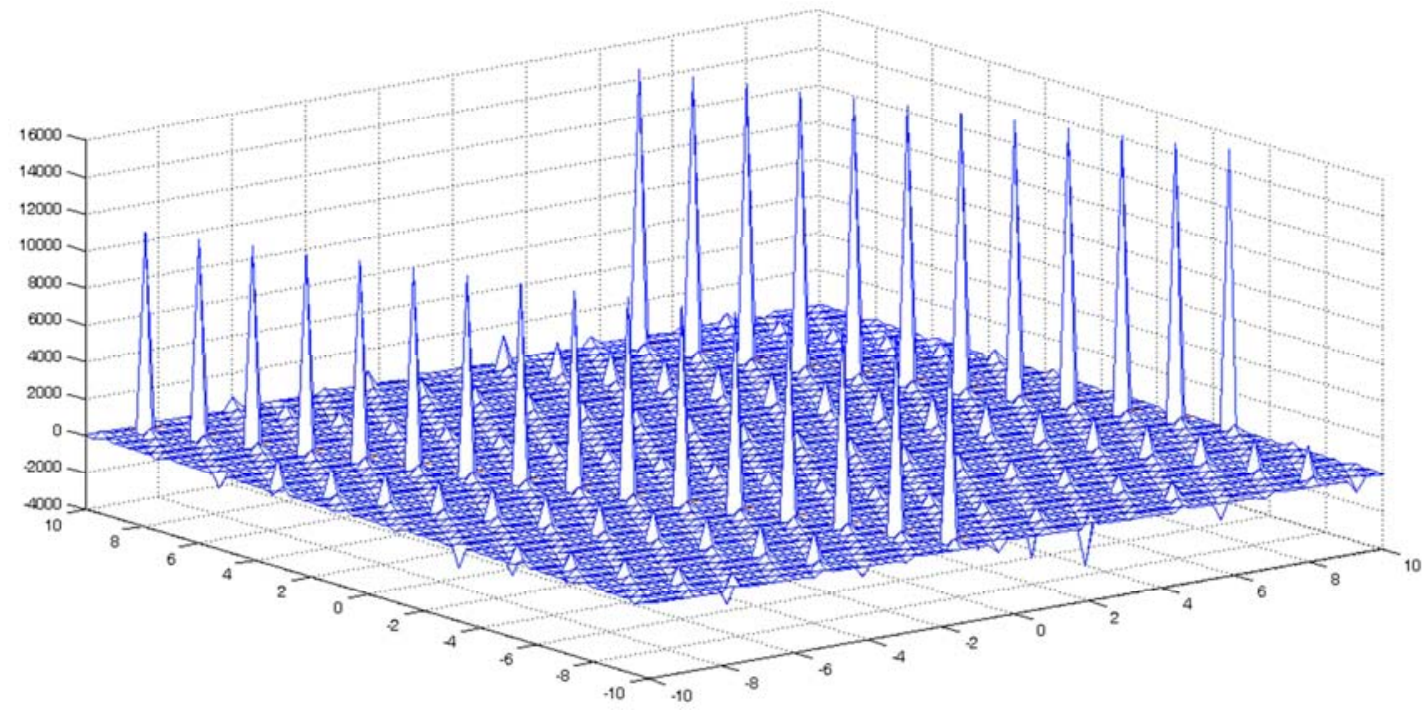

Figure 6. The Graph of $u_{2_{12}}(x, t)$ for $k=8, v=2, q=4, p=2 \& B=0.1$.

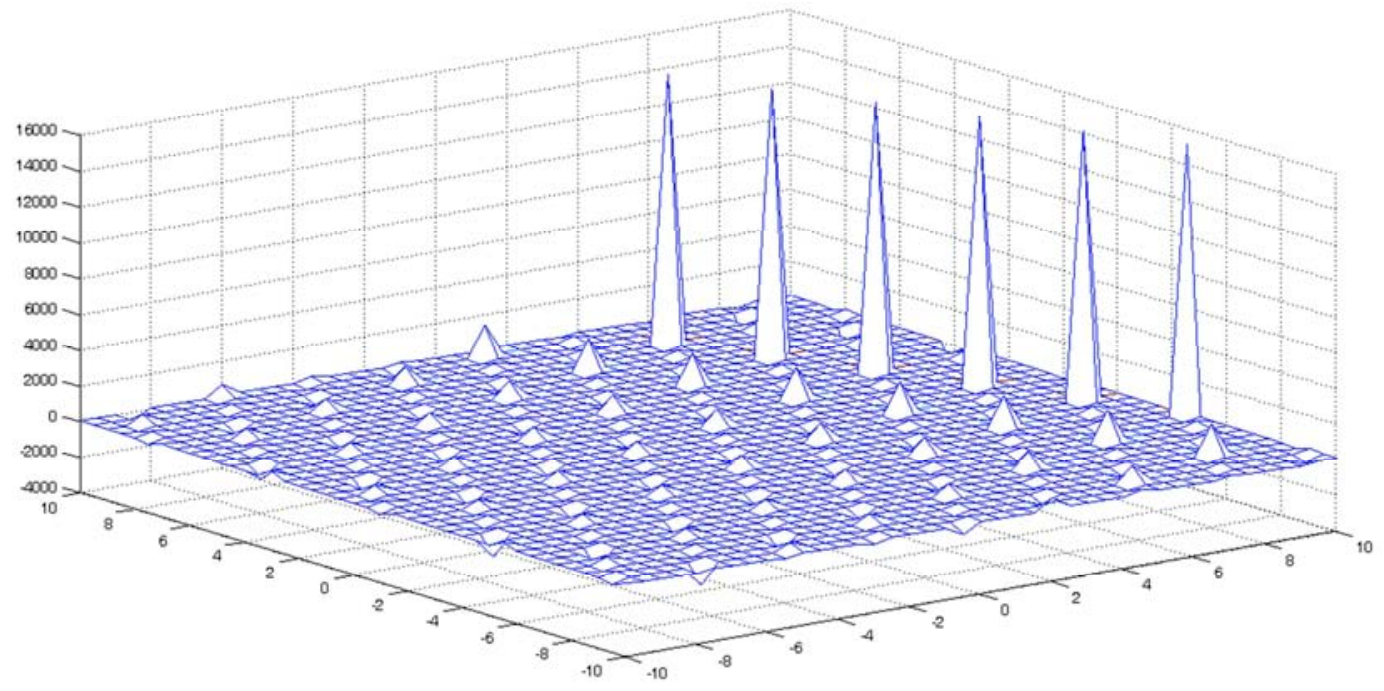

Figure 7. The Graph of $u_{2_{12}}(x, t)$ for $k=8, v=2, q=4, p=2 \& B=0.1$.

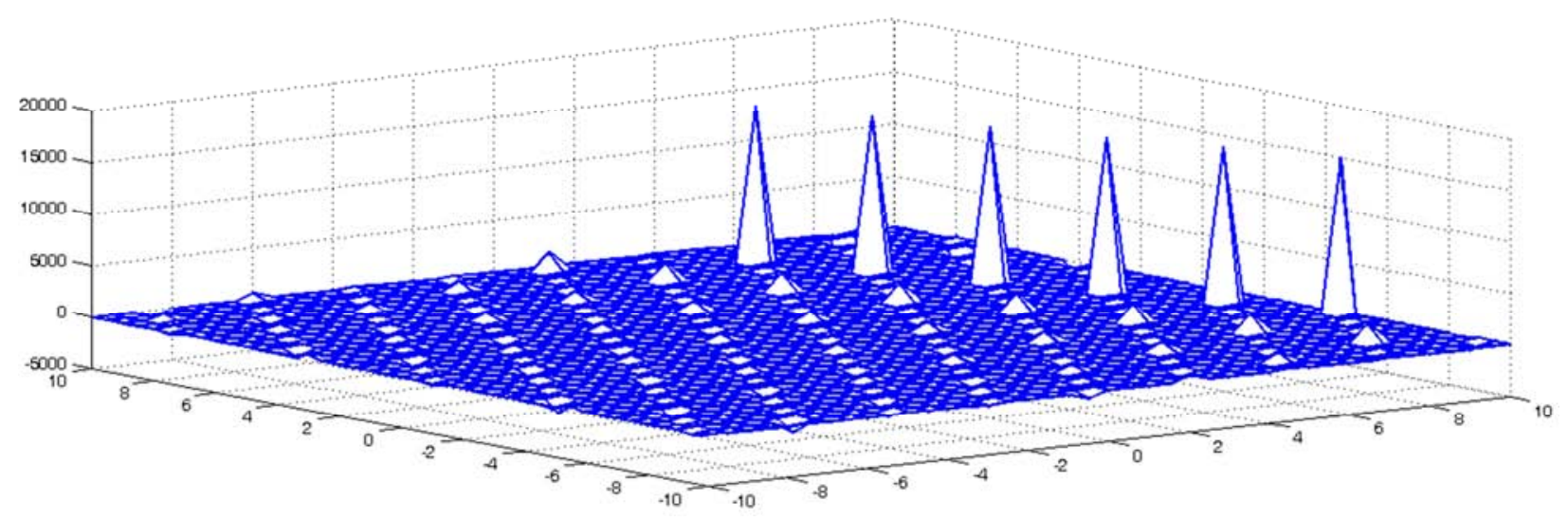

Figure 8. The Graph of $u_{2_{12}}(x, t)$ for $k=8, v=2, q=4, p=2 \& B=0.1$. 


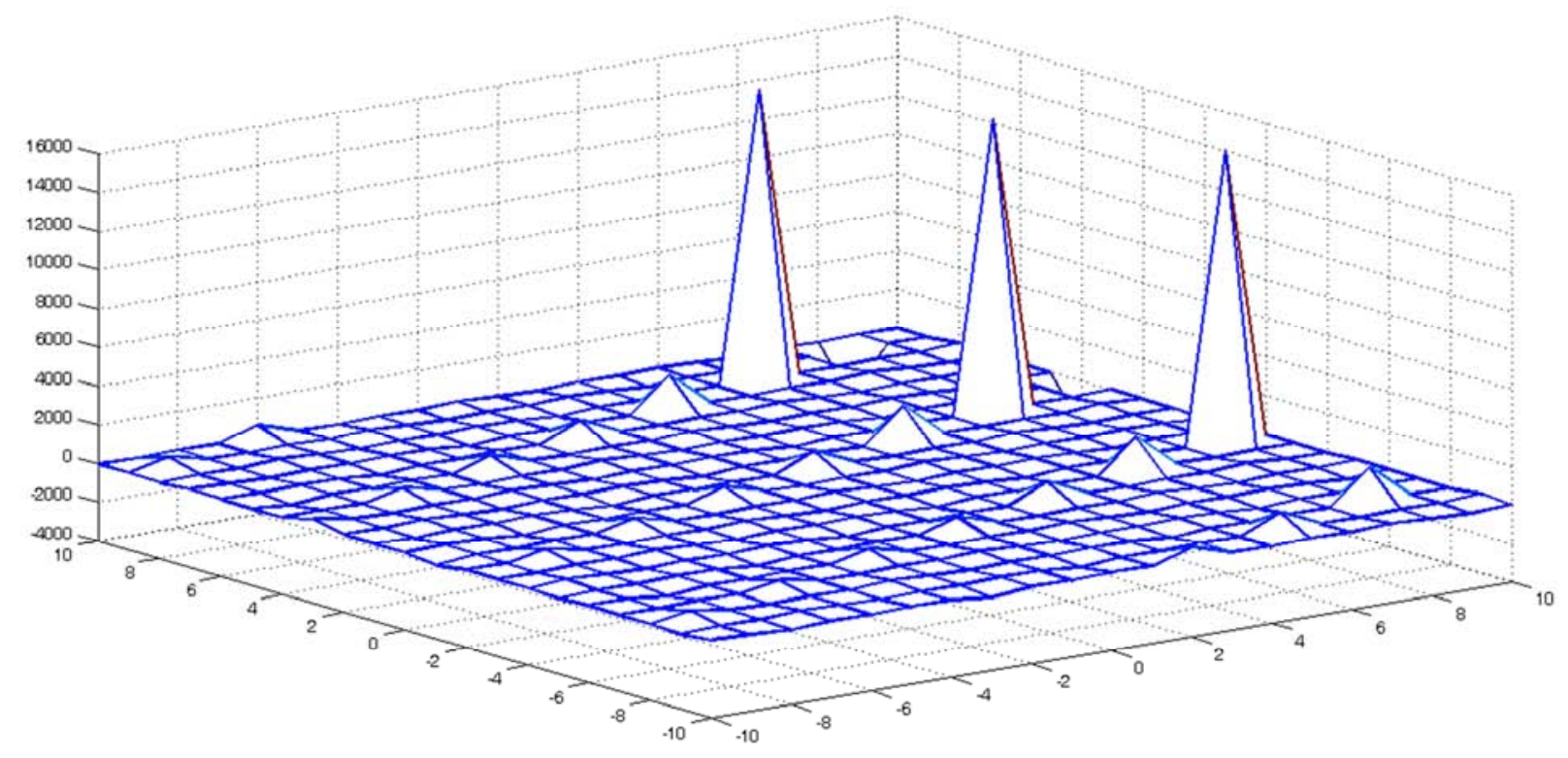

Figure 9. The Graph of $u_{2_{12}}(x, t)$ for $k=8, v=2, q=4, p=2 \& B=0.1$.

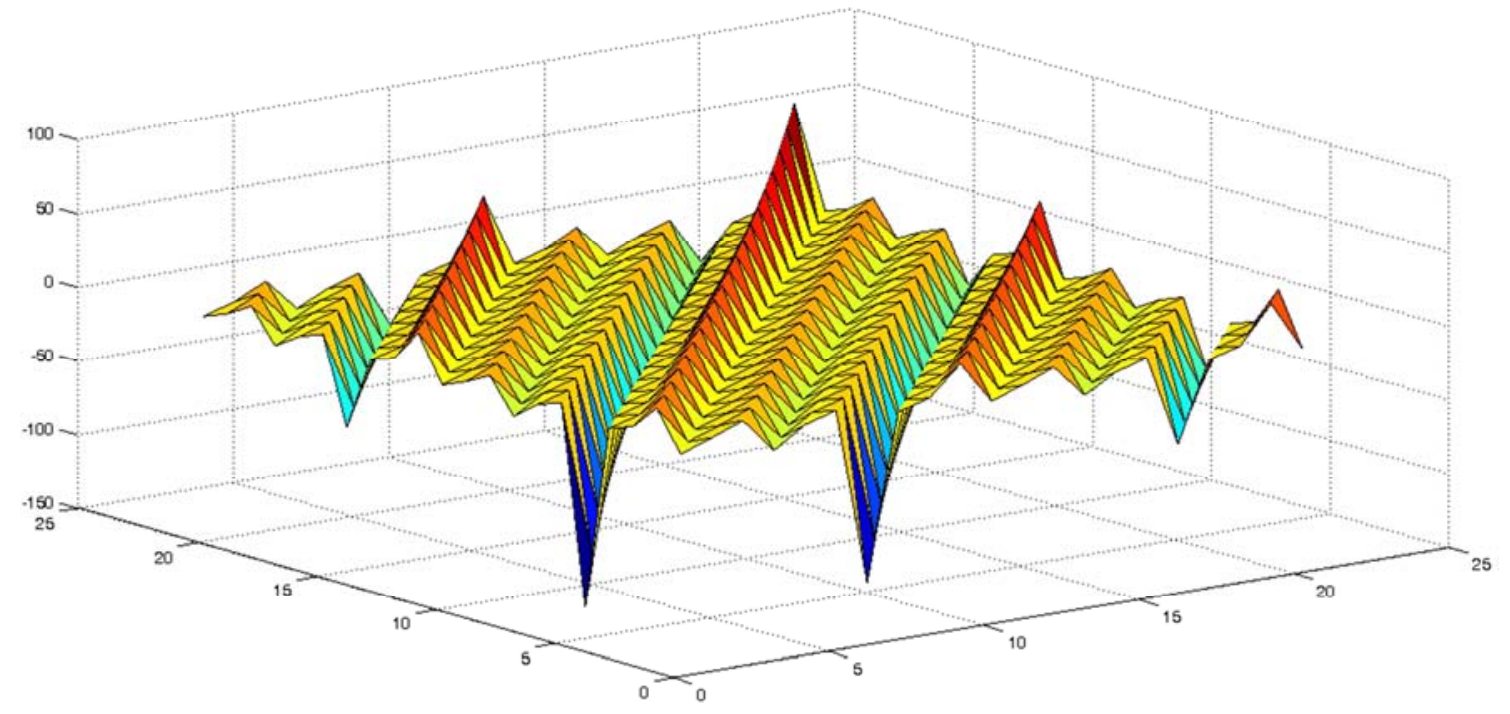

Figure 10. The Graph of $u_{2_{12}}(x, t)$ for $k=8, v=2, q=4, p=2 \& B=0.1$.

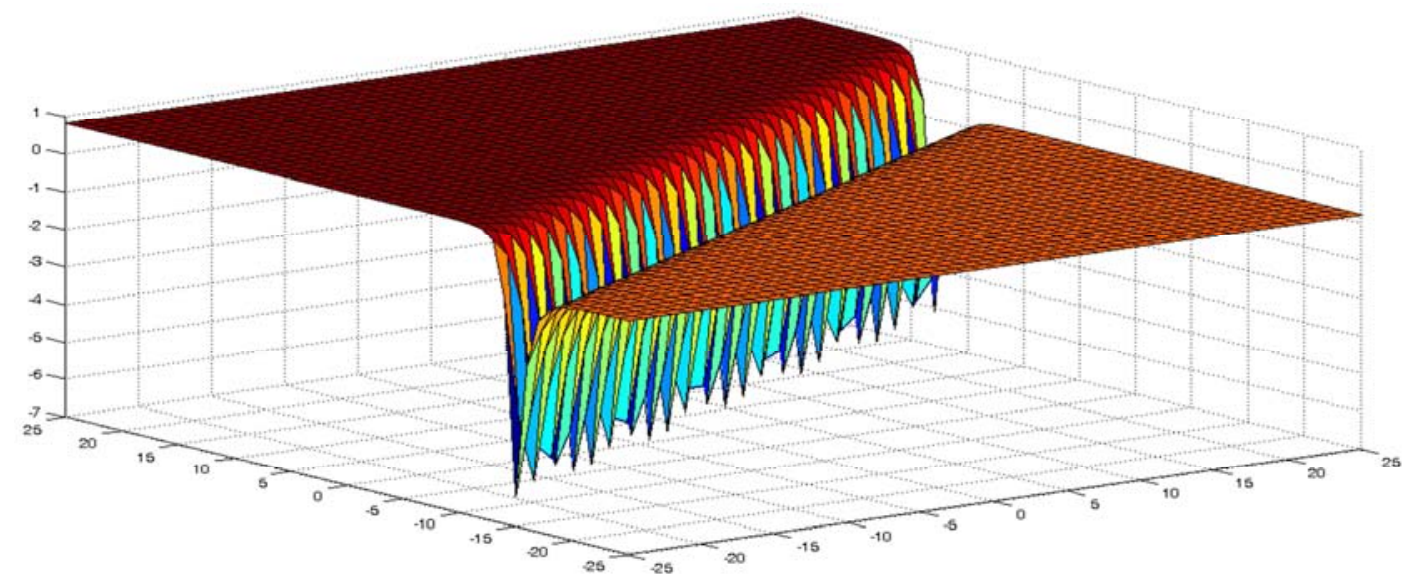

Figure 11. The Graph of $u_{2_{4}}(x, t)$ for $k=-2, v=2, q=1 / 12, p=0.75 \& B=2$. 


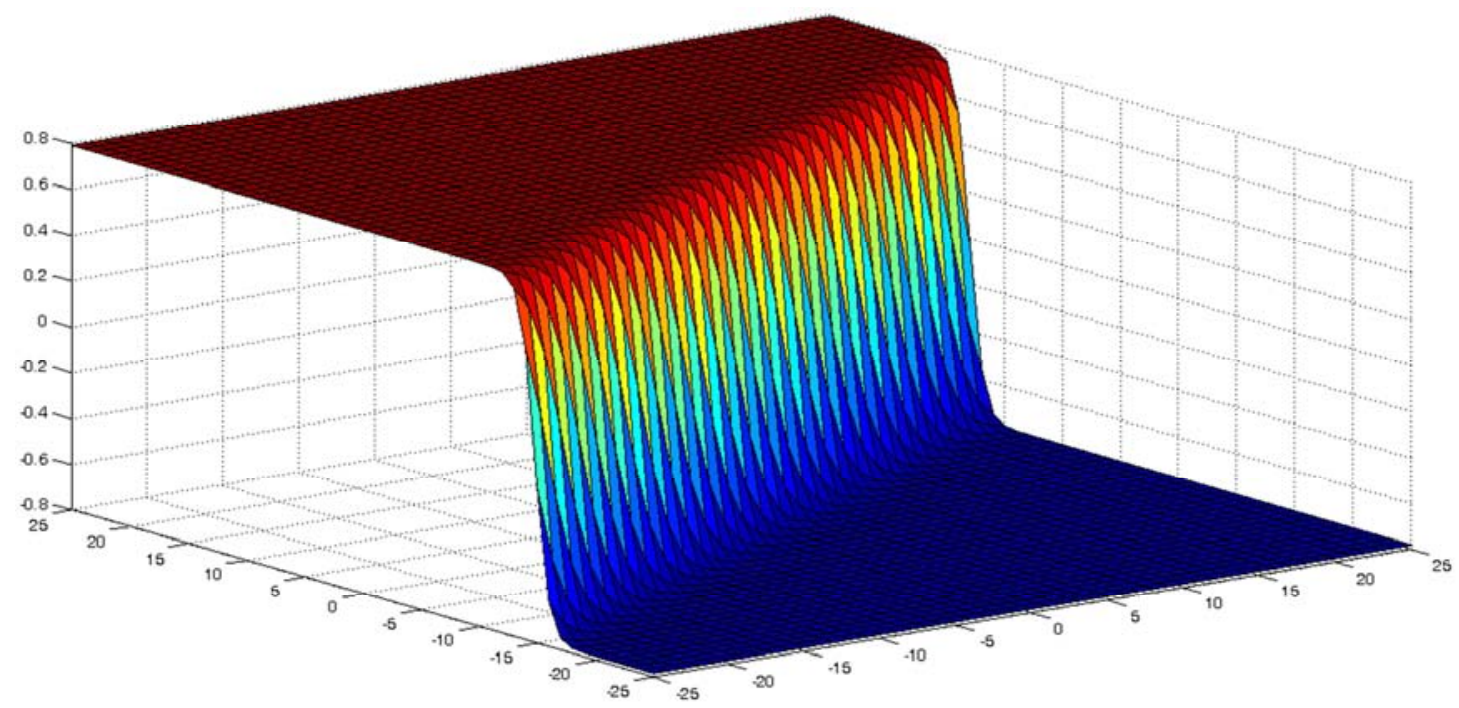

Figure 12. The Graph of $u_{2_{1}}(x, t)$ for $k=-2, v=2, q=1 / 12, p=0.75 \& B=2$.

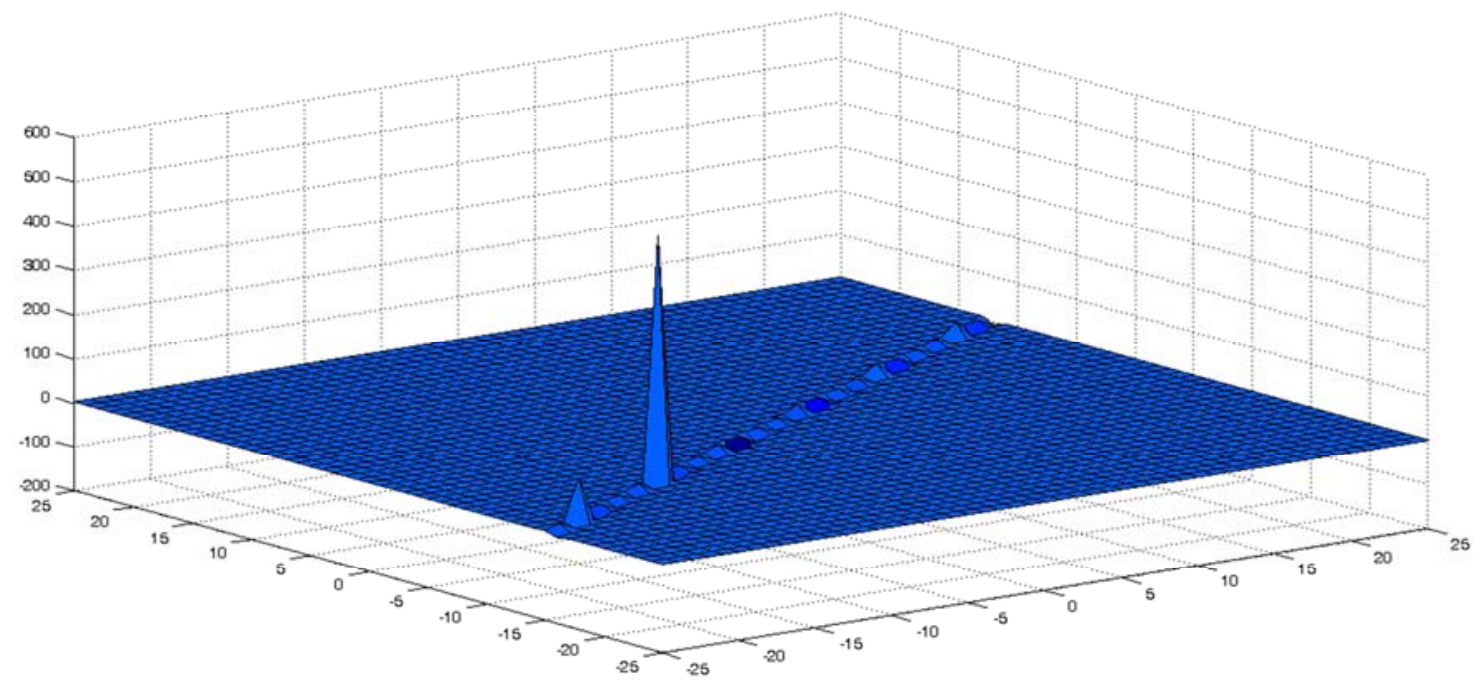

Figure 13. The Graph of $u_{2_{4}}(x, t)$ for $k=-2, v=2, q=1 / 12, p=0.75 \& B=2$.

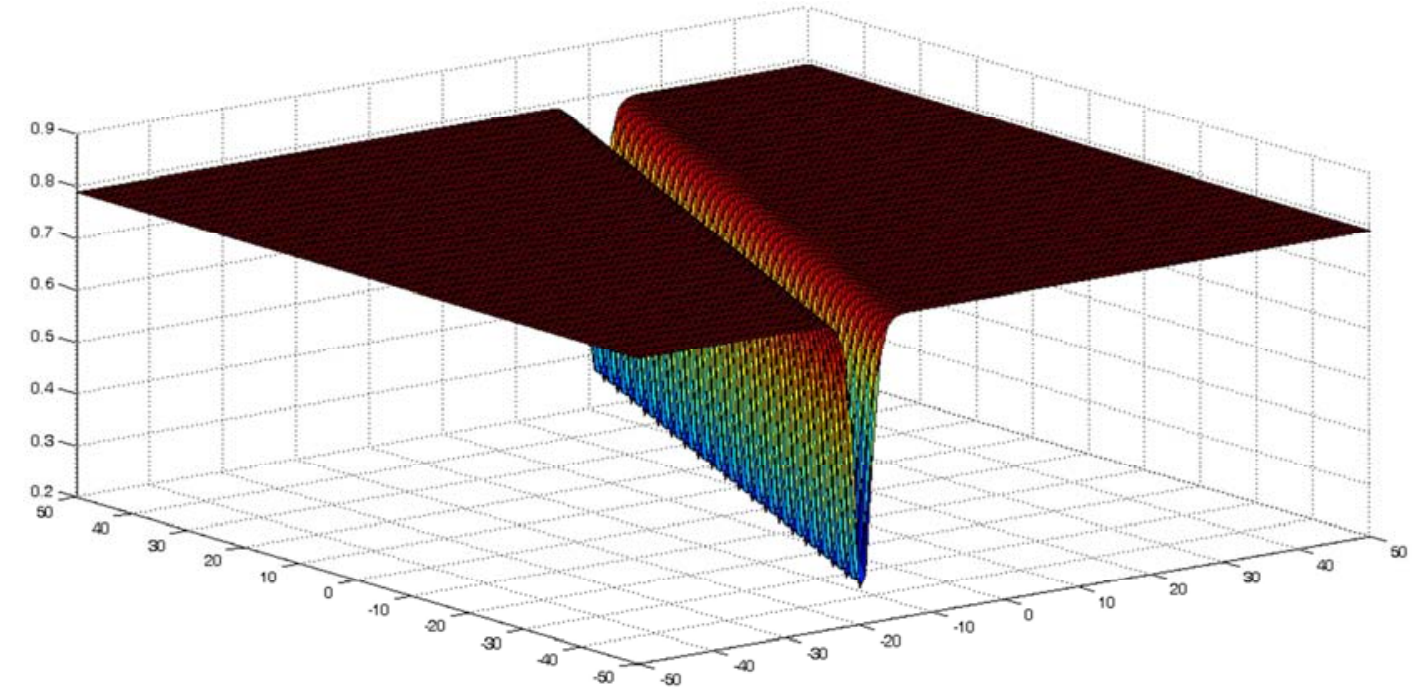

Figure 14. The Graph of $u_{3_{1}}(x, t)$ for $k=-1, v=2, q=0.5, p=2 \& B=3$. 


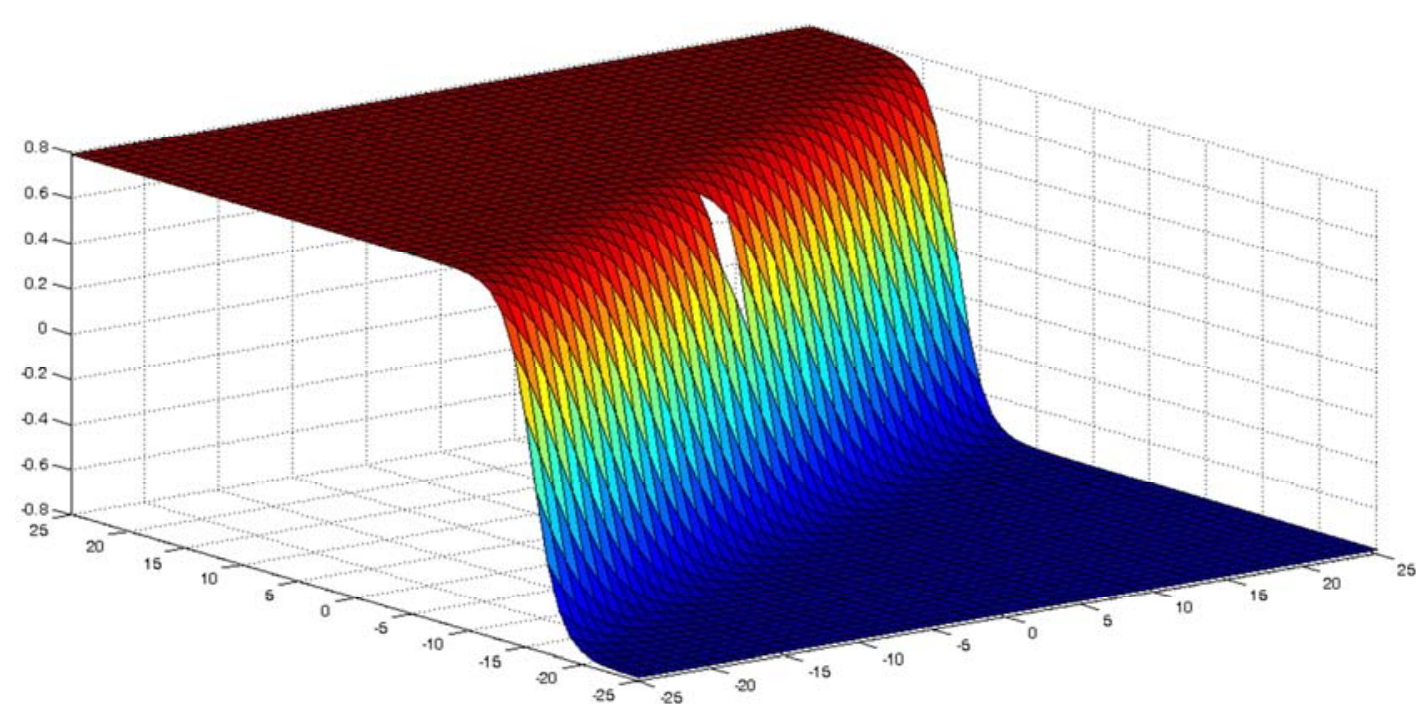

Figure 15. The Graph of $u_{2_{4}}(x, t)$ for $k=-2, v=2, q=1 / 12, p=0.75 \& B=2$.

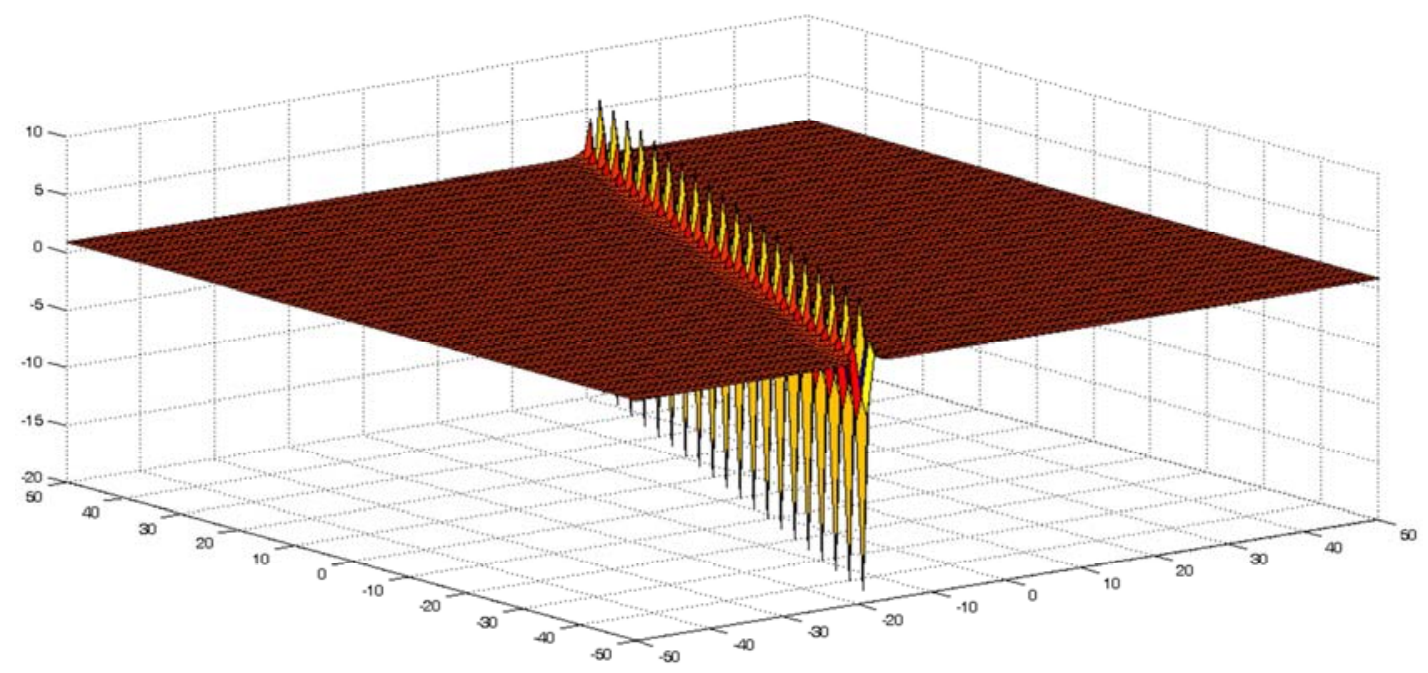

Figure 16. The Graph of $u_{3_{2}}(x, t)$ for $k=-1, v=2, q=0.5, p=2 \& B=3$.

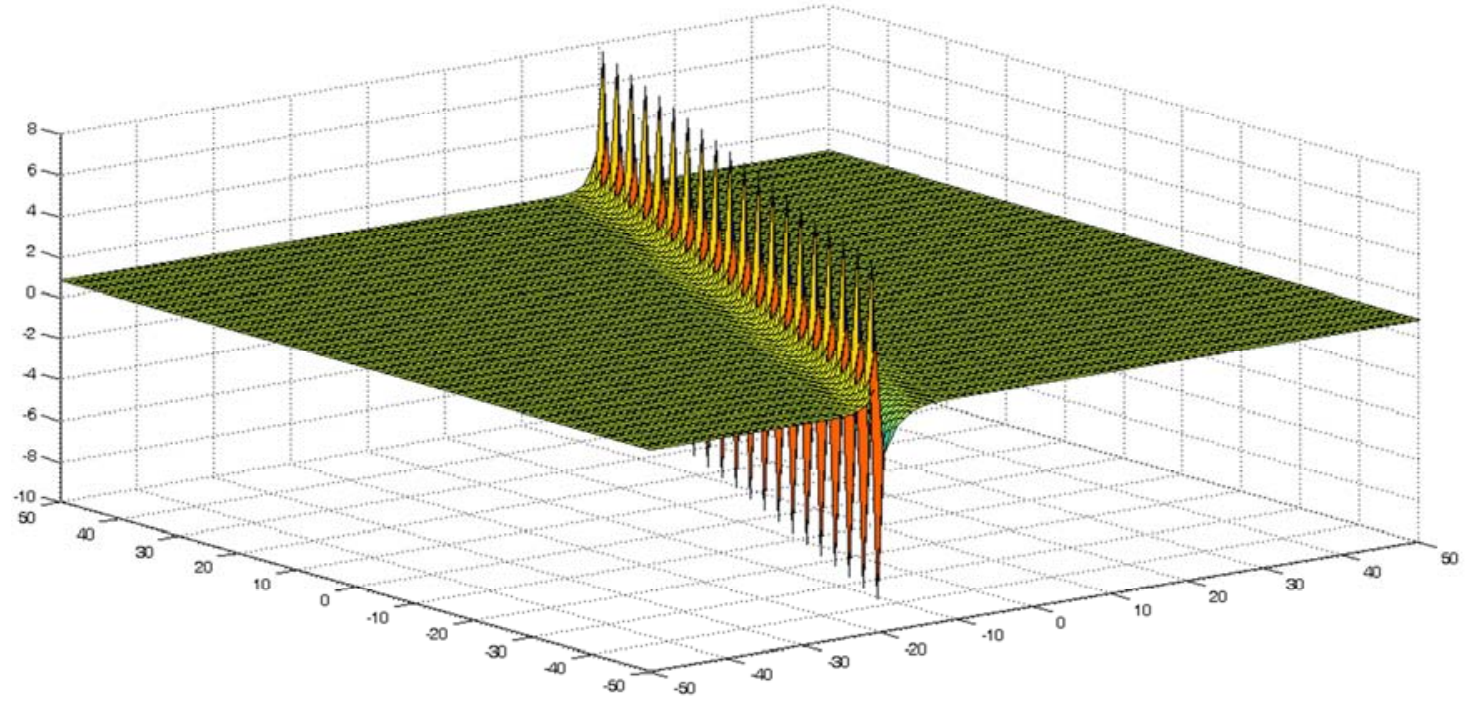

Figure 17. The Graph of $u_{3_{4}}(x, t)$ for $k=-1, v=2, q=0.5, p=2 \& B=3$. 


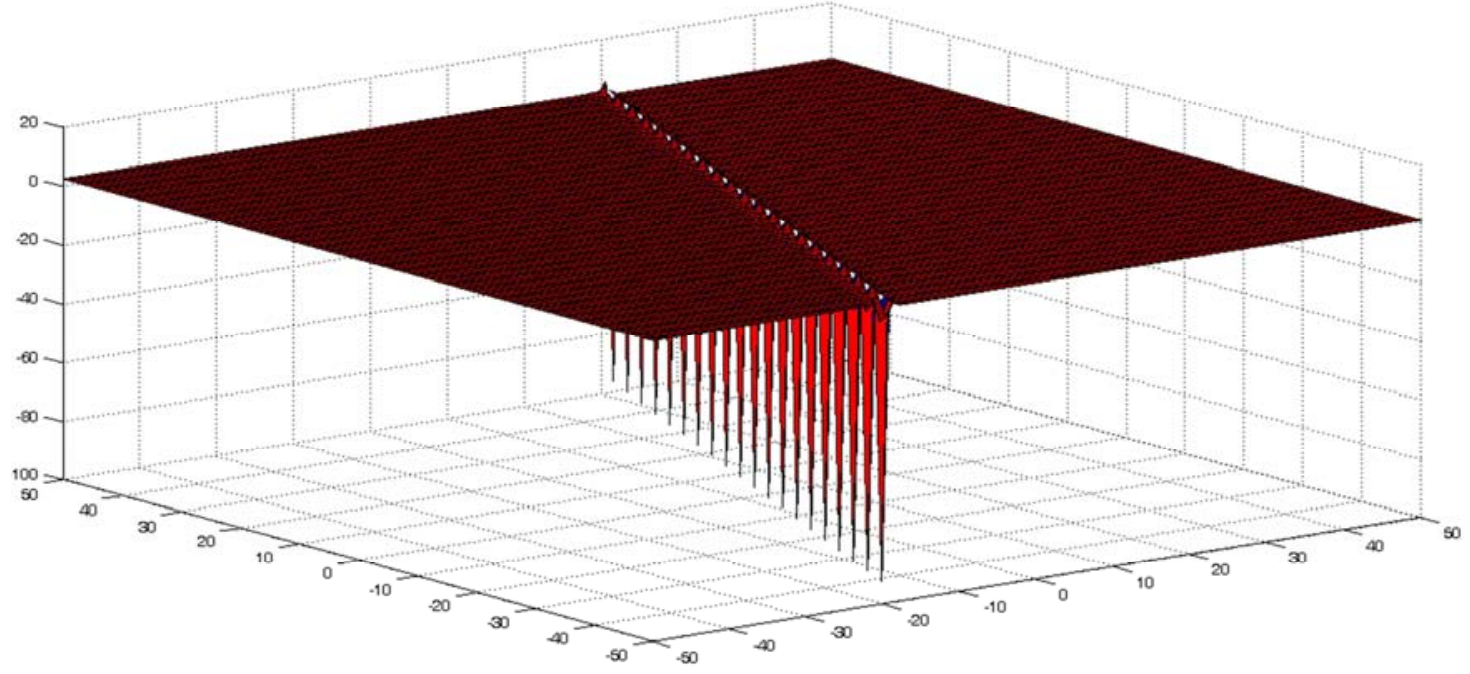

Figure 18. The Graph of $u_{3_{5}}(x, t)$ for $k=-1, v=2, q=0.5, p=2 \& B=3$.

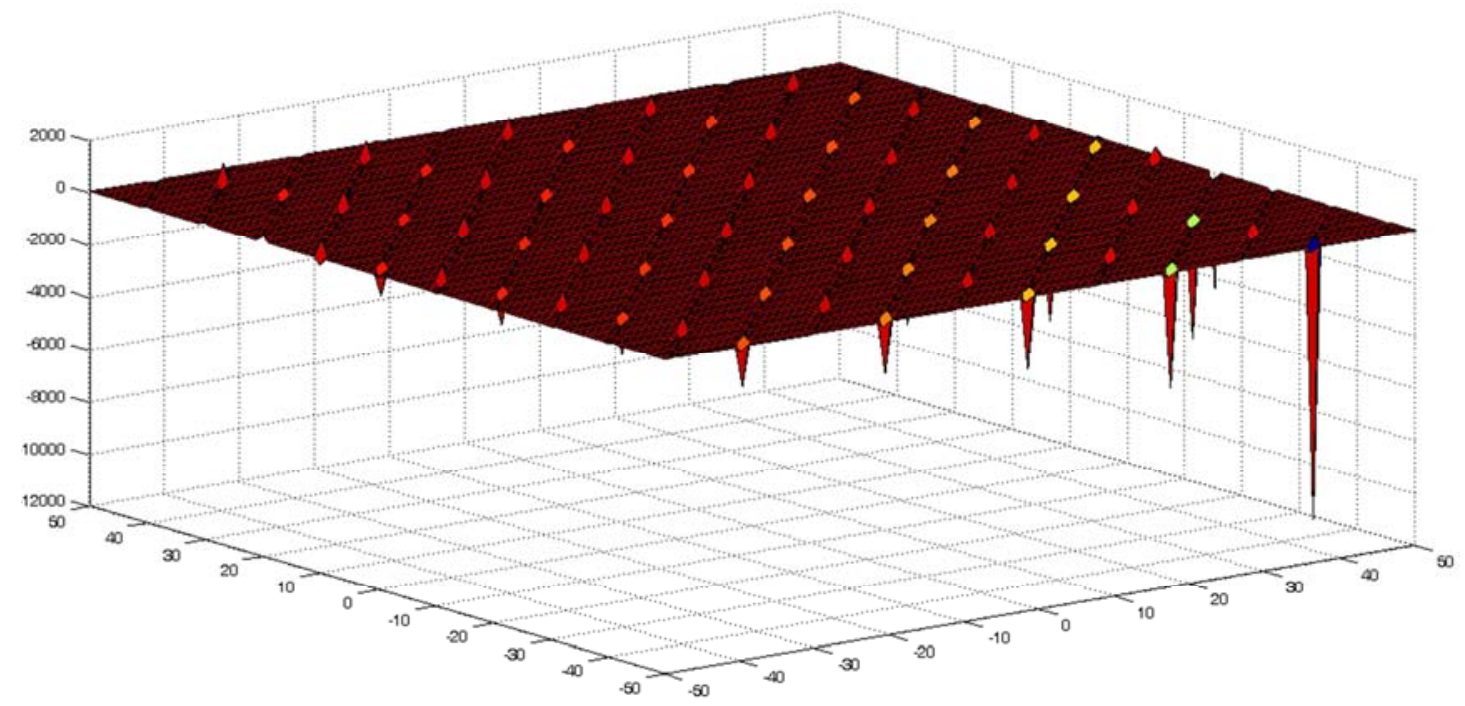

Figure 19. The Graph of $u_{3_{6}}(x, t)$ for $k=-1, v=2, q=0.5, p=2 \& B=3$.

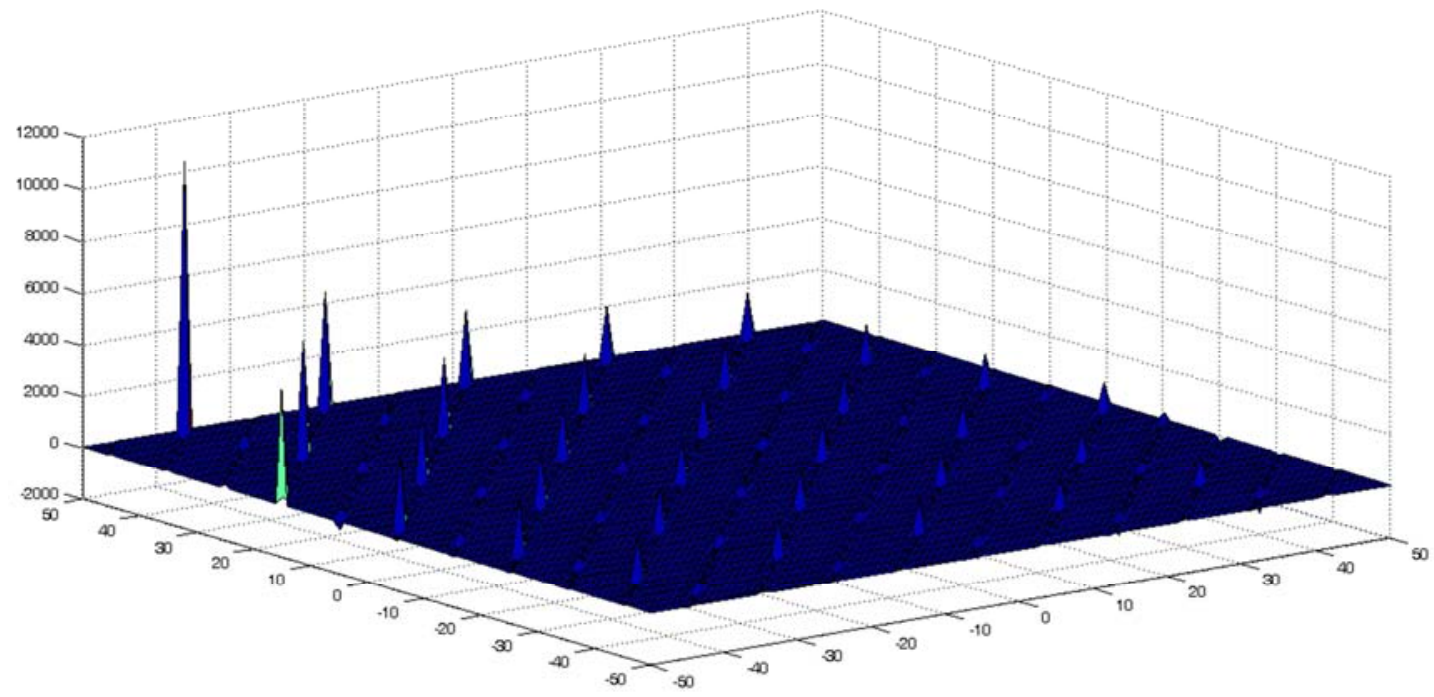

Figure 20. The Graph of $u_{3_{7}}(x, t)$ for $k=-1, v=2, q=0.5, p=2 \& B=3$. 


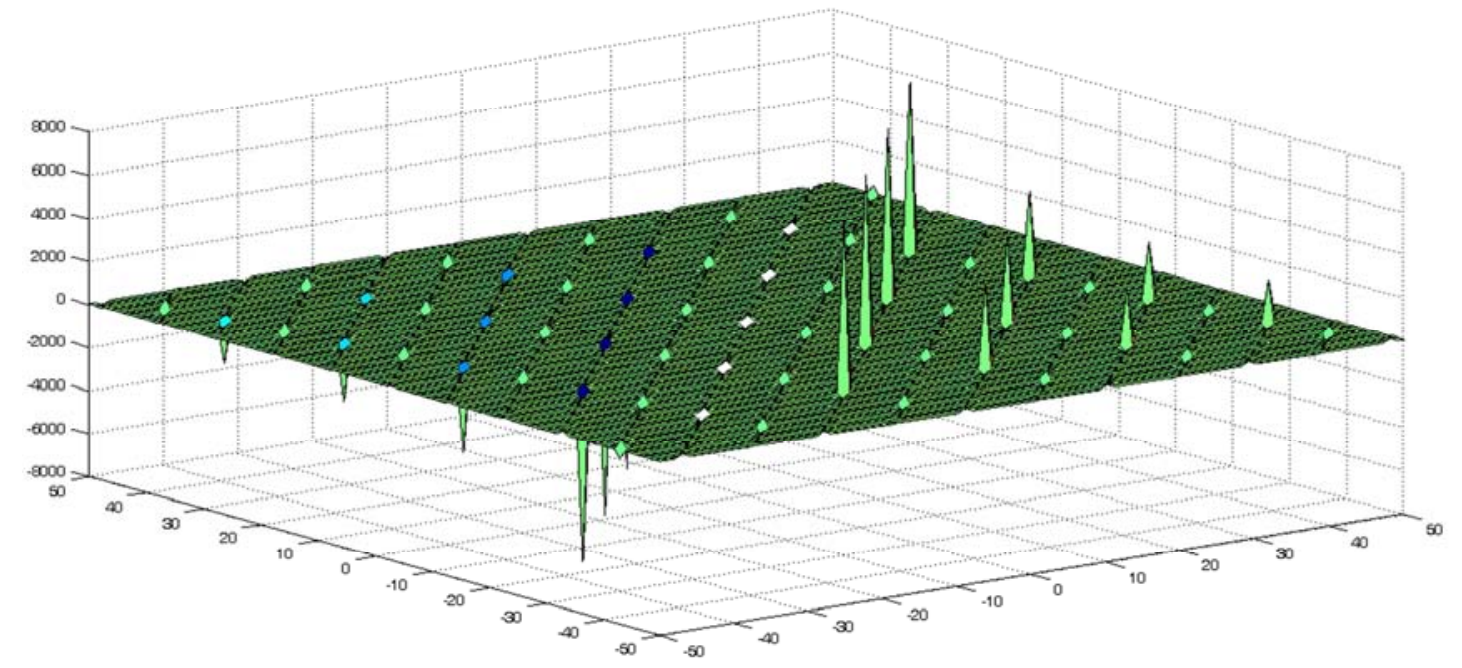

Figure 21. The Graph of $u_{3_{8}}(x, t)$ for $k=-1, v=2, q=0.5, p=2 \& B=3$.

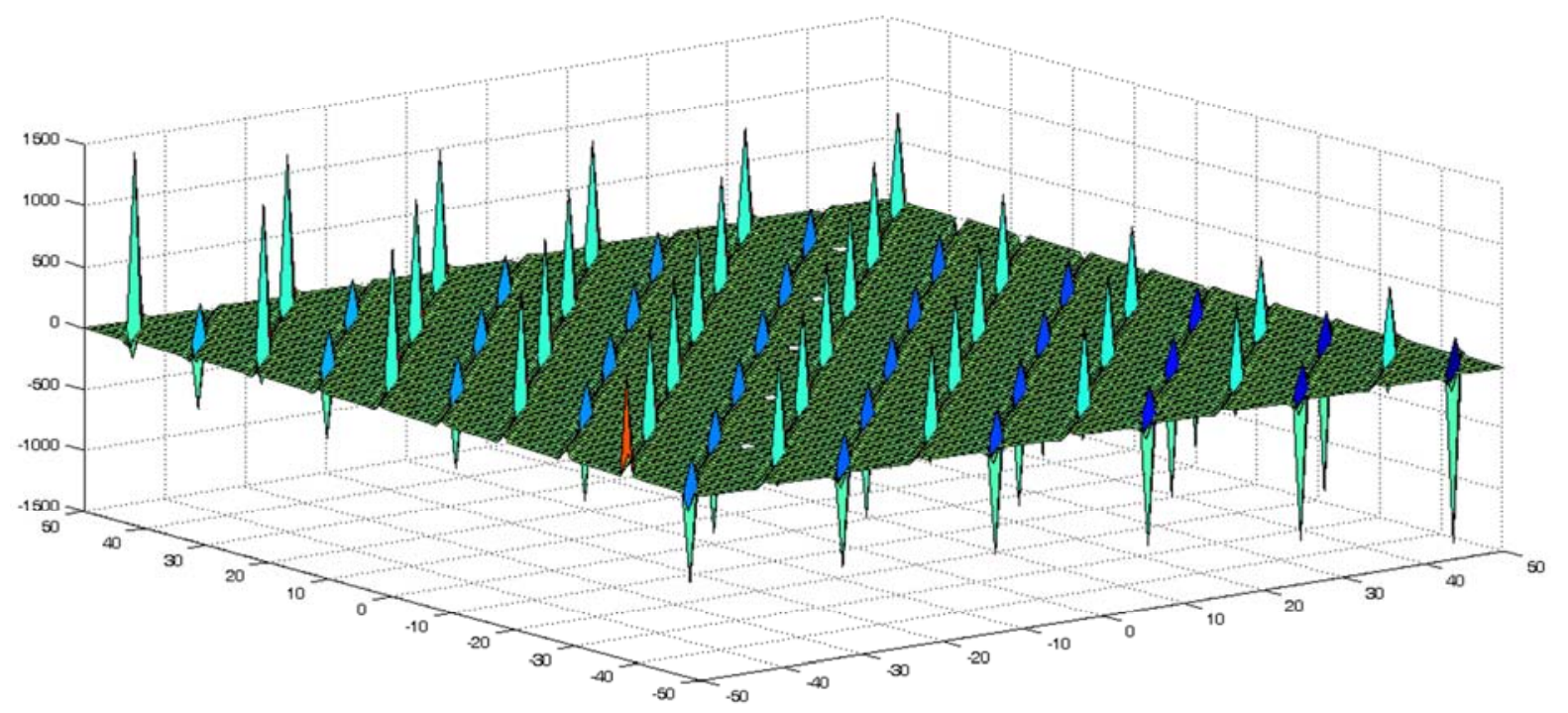

Figure 22. The Graph of $u_{3_{9}}(x, t)$ for $k=-1, v=2, q=0.5, p=2 \& B=3$.

\section{Conclusion}

In this article, we apply the improved $\left(G^{\prime} / G\right)$-expansion method where $G=G(\eta)$ satisfies the second-order nonlinear ordinary differential equation $G G^{\prime \prime}=p G G^{\prime}+q G^{2}+v\left(G^{\prime}\right)^{2}$ to generate more general solution and a rich class of new exact traveling wave solutions of nonlinear PDE, namely, the Simplified Modified Camassa Holm (MCH). As result we obtained more general solution and many new exact traveling wave solutions compared to the result obtained by the improved $\left(G^{\prime} / G\right)$-expansion method and the basic $\left(G^{\prime} / G\right)$ expansion method where $G=G(\eta)$ satisfies the secondorder linear ordinary differential equation, $G^{\prime \prime}+\lambda G^{\prime}+\mu G=0$. The presented solutions may express a variety of new features of waves. Moreover, the obtained exact solutions reveal that the improved $\left(G^{\prime} / G\right)$-expansion method with the second-order nonlinear ordinary differential equation is a promising mathematical tool, because, it can establish abundant new traveling wave solutions with different physical structures. Subsequently, the used method could lead to construct many new traveling wave solutions for various nonlinear PDEs which frequently arise in scientific real time application fields.

\section{References}

[1] C. Rogers and W. F. Shadwick, Backlund Transformations, Academic Press, New York (1982).

[2] He, J. H., Wu, X. H.: Construction of Solitary Solution and Compaction-Like Solution by Variational Iteration Method. Chaos Solitons and Fractals 29, 108-113 (2006).

[3] Hirota, R.: Exact Solution of the Kdv Equation for Multiple Collisions of Solitons. Physical Review Letters 27, 1192-1194 (1971).

[4] Borhanifar, A., Jafari, H., Karimi, S. A.: New solitary wave solutions for the bad Boussinesq and good Boussinesq equations, Numer. Methods for Partial Differential Equations 25, 1231-1237 (2009). 
[5] Borhanifar, A., Jafari, H., Karimi, S. A.: New solitons and periodic solutions for the Kadomtsev-Petviashvili equation. Nonlinear Sci. Appl. 4, 224-229. (2008).

[6] Liu, G. T., Fan, T. Y.: New applications of developed Jacobi elliptic function expansion methods. Phys. Lett. A 345, 161166 (2005).

[7] Wazwaz, A. M.: Exact solutions to the double sinh-Gordan equation by tanh method and a variable separated ode method. Compt. Math. Appl. 501685-1696 (2005).

[8] Malfliet, W., Hereman, W.: The tanh method: exact solutions of nonlinear evolution and wave equations. Phys. Scr. 54, 563-568 (1996).

[9] Wang, M. L.: Exact solution for a compound KdV-Burgers equations. Phys. Lett. A 213, 279- 287 (1996).

[10] Malfliet, W.: Solitary wave solutions of nonlinear wave equations. Am. J. Phy. 60, 650-658 (1992).

[11] Malfliet, W., Hereman, W.: The tanh method: II. Perturbation technique for conservative systems. Phys Scr. 54, 569-575 (1996).

[12] Zhou, X. W., Wen, Y. X., He J. H.: Exp-function method to solve the nonlinear dispersive $\mathrm{k}(\mathrm{m}, \mathrm{n})$ equations, Int. J. Nonlinear Sci. Numer. Simul. 9, 301-306 (2008).

[13] Liu, G. T, Fan, T. Y.: New applications of developed Jacobi elliptic function expansion methods. Phys. Lett. A 345, 161166 (2005).

[14] Ablowitz, M. J., Segur, H.: Solitons and Inverse Scattering Transform. SIAM, Philadelphia (1981).

[15] Wang, M. L., Li, X. Zhang, J.: The (G'/G)-expansion method and traveling wave solutions of nonlinear evolution equations in mathematical physics. Phys. Lett. A 372 417-423 (2008).

[16] Ozis, T. and Aslan, I., "Application of the $\mathrm{G}^{\prime} / \mathrm{G}$-expansion method to Kawahara type equations using symbolic computation," Appl. Math. Computation, 216, 2360-2365, 2010.

[17] Naher, H., Abdullah, F. A. and Akbar, M. A., "The G'/G expansion method for abundant traveling wave solutions of Caudrey-Dodd-Gibbon equation," Math. Prob. Eng., Article ID: 218216, 11 pages, 2011.
[18] Jabbari, A., Kheiri, H. and Bekir, A., "Exact solutions of the coupled Higgs equation and the Miccari system using He's semi-inverse method and $\mathrm{G}^{\prime} / \mathrm{G}$-expansion method," Computers Math. Appli. 62, 2177-2186, 2011.

[19] Naher, H. and Abdullah, F. A., "The basic G'/G -expansion method for the fourth order Boussinesq equation," Appl. Math., 3, 1144-1152, 2012.

[20] Zhang, J. Jiang, F. and Zhao, X., "An improved (G'/G)expansion method for solving nonlinear evolution equations," Int. J. Computer Math., 87, 1716-1725, 2010.

[21] Zhao, Y. M., Yang, Y. J. and Li, W., "Application of the improved (G'/G)-expansion method for the Variant Boussinesq equations," Appl. Math. Sci., 5, 2855-2861, 2011.

[22] Nofel, T. A, Sayed, M., Hamad, Y. S. and Elagan, S. K., "The improved (G'/G)-expansion method for solving the fifth-order KdV equation," Annals of Fuzzy Math. Informatics, 3, 9- 17, 2011.

[23] Naher, H., Abdullah, F. A. and Akbar, M. A., "New traveling wave solutions of the higher dimensional nonlinear evolution equation by the improved (G'/G)-expansion method," World Appl. Sci. J., 16, 11-21, 2012.

[24] Md. Nur Alam and M. Ali Akbar Traveling Wave Solutions of Nonlinear Evolution Equations Via the New Generalized (G'/G)-Expansion Method 1 (4) (2013):129-136, DOI: 10.13189/ujcmj.2013.010403.

[25] Abdollah Borhanifar and Reza Abazari, General Solution of Two Generalized Form of Burgers Equation by Using the (G'/G)-Expansion Method Applied Mathematics, 3 (2012), 158- 168 http://dx.doi.org/10.4236/am.2012.32025.

[26] Abaker A. Hassaballa et al, Applications of the Improved G_/G Expansion Method for Solve Burgers- Fisher Equation. Journal of Computational and Theoretical Nano science Vol. 14, 4664-4668, 2017.

[27] A. M. Wazwaz, Appl. Math. Comput. 163, 1165-1179 (2005). X. Liu, L. Tian and, Y. Wu, Appl. Math. Comput. 217, 1376$1384(2010)$.

[28] Hasibun Naher et al. Some New Solutions of The $(1+1)-$ Dimensional PDE via The Improved ( $G$ '/G)-Expansion Method. 